\title{
Dietary flaxseed oil rich in omega-3 suppresses severity of type 2 diabetes mellitus via anti-inflammation and modulating gut microbiota in rats
}

Lili Zhu ${ }^{1 \dagger}$, Liping Sha ${ }^{2 \dagger}$, Ke Li ${ }^{3}$, Zhen Wang ${ }^{4}$, Ting Wang ${ }^{1}$, Yiwei Li ${ }^{1}$, Ping Liư ${ }^{2}$, Xiaoying Dong ${ }^{2}$, Youping Dong ${ }^{2}$, Xiaoxia Zhang ${ }^{5^{*}}$ and Hao Wang ${ }^{1 *}$ (D)

\begin{abstract}
Background: Type 2 diabetes mellitus (T2DM) is closely associated with hyperglycemia, abnormal lipid profiles, chronic low-grade inflammation and gut dysbiosis. Dietary intervention plays a crucial role in the control of diabetes. Flaxseed oil (FO), a plant-derived omega-3 ( $\omega-3)$ polyunsaturated fatty acids (PUFAs), is rich in a-linolenic acid (ALA) which has been proved to benefit for chronic metabolic disease. However, the exact effects of dietary FO on T2DM remains largely unclear.
\end{abstract}

Methods: In the present study, SD rats were randomly allocated into four groups: pair-fed (PF) with corn oil (CO) group (PF/CO); DM with CO group (DM/CO); PF with FO group (PF/FO); DM with FO group (DM/FO). A diabetic rat model was generated by a single intraperitoneal injection of streptozotocin-nicotinamide (STZ-NA). After 5 weeks of intervention, rats were euthanized and associated indications were investigated.

Results: Dietary FO significantly reduced fasting blood glucose (FBG), glycated hemoglobin (GHb), blood lipid, plasma lipopolysaccharide (LPS), interleukin (IL)-1 $\beta$, tumor necrosis factor (TNF)-a, IL-6, IL-17A and malondialdehyde (MDA), compared to control group, respectively. Moreover, body mass (BM) and superoxide dismutase (SOD) in DM/FO group were dramatically increased respectively, compared with those in DM/CO group. But insulin (INS) and homeostasis model assessment of insulin resistance (HOMA-IR) remained no significant difference between DM/CO group and DM/FO group. Sequencing analysis of gut microbiota showed a reduction in the relative abundance of Firmicutes and Blautia, as well as a reduction in the ratio of Bacteroidetes-Firmicutes in DM/FO group compared to DM/CO group. An elevation in the relative abundance of Bacteroidetes and Alistipes were detected in DM/FO group. Acetic acid, propionic acid and butyric acid belonging to short chain fatty acids (SCFAs) as gut microbiota metabolites, were dramatically increased after FO intervention. Correlation analysis revealed that the relative abundance of Firmicutes and Blautia were positively correlated with IL-1 $\beta$, TNF- $a$, IL-6, IL-17A or LPS, respectively. Additionally, Bacteroidetes and Alistipes were negatively correlated with LPS.

Conclusions: Taken together, dietary FO ameliorated T2DM via suppressing inflammation and modulating gut microbiota, which may potentially contribute to dietary control of diabetes.

Keywords: Flaxseed oil, T2DM, Anti-inflammation, Gut microbiota

\footnotetext{
*Correspondence: zxx1216@163.com; wanghaograduate@126.com

${ }^{\dagger}$ Lili Zhu and Liping Sha contributed equally to this work.

${ }^{5}$ College of Traditional Chinese Medicine, Ningxia Medical University,

Yinchuan 750004, Ningxia, China

${ }^{1}$ Department of Pathogenic Biology and Medical Immunology, School of

Basic Medical Sciences, Ningxia Medical University, Yinchuan 750004, Ningxia,

China

Full list of author information is available at the end of the article
}

(c) The Author(s). 2020 Open Access This article is distributed under the terms of the Creative Commons Attribution 4.0 International License (http://creativecommons.org/licenses/by/4.0/), which permits unrestricted use, distribution, and reproduction in any medium, provided you give appropriate credit to the original author(s) and the source, provide a link to the Creative Commons license, and indicate if changes were made. The Creative Commons Public Domain Dedication waiver (http://creativecommons.org/publicdomain/zero/1.0/) applies to the data made available in this article, unless otherwise stated. 


\section{Introduction}

Prevalence of T2DM presents an observably global public health problem. According to statistical data of the International Diabetes Federation, more than 450 million people suffered from diabetes worldwide in 2017, and this number was expected to hit 629 million by 2045 [1]. Thus, novel strategies for the control of T2DM are urgently needed.

Gut microbiota represents a complicated community involving bacteria in the gastrointestinal tract, which usually maintains a mutually relationship with its host. Numerous studies have demonstrated that the pathogenesis of T2DM is closely related with gut microbiota $[2,3]$. It has been proven that diabetic individuals possess a high quantity of gram-negative bacteria, especially those belonging to the phylum Proteobacteria [4]. In a rat model of T2DM, the proportion of Firmicutes was increased, while the abundance of Bacteroides was decreased [5]. Regulation of gut microbiota may improve metabolism in T2DM [6].

In T2DM, gut microbiota dysbiosis promotes generation of lipopolysaccharide (LPS) triggering chronic lowdegree inflammation by binding to toll-like receptor 4 (TLR-4) [7]. Excessive translocation of gut-derived LPS to the liver via portal circulation can subsequently promote the inflammatory cascade, leading to the release of inflammatory cytokines involving interleukin (IL)-1 $\beta$, IL6 , IL-17A and tumor necrosis factor (TNF)- $\alpha$ [8]. In addition to LPS, other metabolites (such as short chain fatty acids, SCFAs) of gut microbiota have been demonstrated to target multiple pathways in intestine, liver, pancreas and peripheral blood circulation, resulting in the improvement of gut homeostasis, glycemic control, lipids profile, insulin resistance and inflammation [9]. Patients with diabetes exhibited lower contents of SCFAs and the proportion of SCFAs-producing gut bacterial communities including Bifidobacterium, Bacteroidetes and Lactobacillus [10]. At present, SCFAs have been recognized as potential mediators involved in intestinal immune function.

Over the past few decades, human's lifestyle has changed greatly, with increasing animal food consumption and dietary fat intake. Incidence of T2DM was positively related to these unhealthy lifestyles, indicating that dietary factors play a crucial role in the onset and progression of T2DM [11]. One of the most common dietary approaches against T2DM is the increase in the consumption of omega-3 ( $\omega-3)$ polyunsaturated fatty acids (PUFAs) [12, 13]. A survey has suggested that the low incidence of diabetes in Greenland Eskimos may partly due to high consumption of fish oil which is rich in $\omega-3$ PUFAs, especially eicosapentaenoic acid (EPA) and docosahexaenoic acid (DHA) [14]. Flaxseed oil (FO), a plant source of $\omega-3$ PUFAs, has shown to benefit to chronic metabolic, such as diabetes [15]. The advantage of FO over fish oil is that the food sources are less expensive and are free of heavy metal contamination. In addition, FO has been demonstrated to suppress the production of TNF- $\alpha$, IL- 6 and IL- $1 \beta[16,17]$. Accumulating studies have shown that FO protects against inflammation [8] and oxidative stress [18] in metabolic disease models. However, the relationships among dietary FO, chronic inflammation and gut microbiota in diabetic rats still remains largely unclear.

The present study aimed to assess the effects of dietary FO and mechanisms related to inflammation and gut microbiota in diabetic rats. Our study may contribute to further understanding of the complicated interaction between diet, the gut microbiota and inflammation of T2DM.

\section{Materials and methods Animals}

Male Sprague-Dawley (SD) rats (200-250 g) were purchased from Central Animal House of NingXia Medical University. Rats were conditioned in standard polypropylene cages (4 rats/cage) with controlled temperature $\left(22 \pm 2{ }^{\circ} \mathrm{C}\right)$, humidity $(40-70 \%)$ and $12 \mathrm{~h}$ light/12 $\mathrm{h}$ darkcycle for 1 week before the experiment. All animal experiments were approved by Ethics Committee of the General Hospital of Ningxia Medical University (No. 2016-232). During the whole experimental period, animals were fed with a balanced commercial diet and water ad libitum. All diets for rats feeding were purchased from TROPHIC Animal Feed High-tech Co., Ltd., Nantong, China.

\section{Diabetic rats}

The rats were fasted $12 \mathrm{~h}$ before the streptozotocin (STZ)-nicotinamide (NA) injection. T2DM was induced by the intraperitoneal (i.p) administration of a single dose $(65 \mathrm{mg} / \mathrm{kg} \mathrm{BM})$ of STZ freshly dissolved in $0.1 \mathrm{M}$ citrate buffer $(\mathrm{pH}=4.5), 15$ min after i.p administration of $110 \mathrm{mg} / \mathrm{kg}$ BM of NA dissolved in normal saline. Animals were allowed to drink $20 \%$ glucose solution overnight to overcome drug-induced hypoglycemia. Rats in the pair-fed (PF) group were i.p injected with an equal volume of citrate buffer and normal saline. Hyperglycemia was confirmed according to the levels of fasting blood glucose (FBG), which was determined in day 3 and 7 after injection. Rats with FBG levels above 13.9 $\mathrm{mmol} / \mathrm{L}$ were considered to T2DM in the experiment [19], followed by feeding various types of diets.

\section{Experimental design}

Rats were randomly divided into four groups (8 rats/ group): (a) PF with corn oil (CO) group (PF/CO), PF rats were fed 10\% w/w CO diet as CO control; (b) DM with $\mathrm{CO}$ group (DM/CO), DM rats were fed $10 \% \mathrm{w} / \mathrm{w} \mathrm{CO}$ diet; (c) PF with flaxseed oil (FO) group (PF/FO), PF rats were fed $10 \%$ w/w FO diet as FO control; (d) DM with 
FO group (DM/FO), DM rats were fed 10\% w/w FO diet. The standard rodent chow pellets were powdered and mixed with FO and re-pelleted so as to contain either $10 \% \mathrm{w} / \mathrm{w}$. Animals from PF/FO group and DM/FO group received $10 \% \mathrm{w} / \mathrm{w}$ FO diet. As control, PF/CO group and DM/CO group received 10\% w/w CO diet. The fatty acid composition of dietary fats are shown in (Additional file 1: Table S1). Feeds were prepared every week and packed sealed bags in quantities sufficient for 1 day's feed. Feeds in the plastic bags were flushed with nitrogen, sealed, and stored at $-20^{\circ} \mathrm{C}$. Feeds which were not consumed by animals were discarded daily. During the experimental period, body mass (BM) and FBG were determined weekly to reflect the alteration of basic indicators. After 5 weeks of feeding, rats were euthanized by $4 \%$ sodium pentobarbital and associated indications were investigated. Blood samples were respectively collected with or without ethylene diamine tetraacetic acid (EDTA) and centrifuged $(700 \times \mathrm{g}$ for $10 \mathrm{~min})$ to obtain plasma or serum samples. Serum samples were used to detect oxidative markers and plasma samples were used to determine the remaining chemical indicators, both of which were stored in $-80^{\circ} \mathrm{C}$ before further analysis.

\section{Plasma biochemical tests}

At the end of the experiment, glycated hemoglobin (GHb) was measured using the Glycosylated Hemoglobin (GHb) enzyme linked immunosorbent assay (ELISA) Kit (Wuhan ColorfulGene Biological Technology Co., Ltd., Wuhan, China). Plasma insulin concentration (INS) was analyzed using commercial enzyme-linked immunoassay kit (Qiaoyi Biology, Anhui, China). Homeostasis model assessment of insulin resistance (HOMA-IR) was calculated as [glucose $(\mathrm{mmol} / \mathrm{L}) \times$ insulin $(\mathrm{mU} / \mathrm{L})] / 22.5$. Total cholesterol $(\mathrm{TC})$, triglyceride (TG), low density lipoprotein (LDL) and high density lipoprotein (HDL) levels in each group were respectively determined using AU400 automatic biochemical analyzer (Olympus, Japan).

\section{Oxidative indicators}

Malondialdehyde (MDA) assay kits and total superoxide dismutase (SOD) activity in each group were respectively determined with thiobarbituric acid (TBA) and hydroxylamine method according to the manufacturer's instructions (Nanjing Jiancheng Bioengineering Ins., Nanjing, China).

\section{Determination of plasma LPS}

Plasma LPS levels of each group were determined using limulus amebocyte lysate kit (Xiamen Bioendo Technologky Co. Ltd., Xiamen, China). Briefly, $50 \mu \mathrm{L}$ of plasma was dispensed to each well in a 96-well plate. At the initial time point, $50 \mu \mathrm{L} /$ well of the limulus amebocyte lysate reagent was added respectively. The plate was incubated at $37^{\circ} \mathrm{C}$ for $30 \mathrm{~min}$. Then $100 \mu \mathrm{L}$ of chromogenic substrate warmed to $37^{\circ} \mathrm{C}$ was added to each well. Incubation was extended for an additional $6 \mathrm{~min}$ at $37^{\circ} \mathrm{C}$. The reaction was stopped by adding $100 \mu \mathrm{L}$ of a $25 \%$ solution of glacial acetic acid. Optical density at $545 \mathrm{~nm}$ was measured with a microplate reader (Thermo Scientific, USA).

\section{ELISA assays}

Plasma inflammatory cytokines including IL- $1 \beta$, TNF- $\alpha$, IL-6, IL-17A and IL-10 were measured by ELISA kits according to the manufacturer's instructions (Shanghai Jianglai Biotech, Shanghai, China). Optical density was measured at $450 \mathrm{~nm}$ within $15 \mathrm{~min}$, using an automated microplate reader (Thermo Fisher Scientific, USA).

\section{Gut microbiota analysis}

The fecal microbial 16S rRNA high throughput sequencing and analysis were performed according to previous studies [3]. After treatment, 5 rats were randomly selected from each group and transferred to fresh sterilized cages. The feces of each rat were respectively collected and immediately stored at $-80^{\circ} \mathrm{C}$ for subsequent DNA extraction.

Total genome DNA from samples was extracted using cetyltrimethylammonium bromide (CTAB) method as previously described [20], which was monitored by electrophoresis on 1\% agarose gels (Additional file 2: Figure S1). According to the concentration, DNA was diluted to $1 \mathrm{ng} / \mu \mathrm{L}$ using sterile water.

The hypervariable V3 to V4 regions of the 16S rRNA gene were amplified with barcoded primers: 341F 5' CCTAYGGGRBGCASCAG-3' and 806R 5'- GGACTA CNNGGGTATCTAAT-3'. All PCR reactions were carried out in $30 \mu \mathrm{L}$ reactions with $15 \mu \mathrm{L}$ of Phusion ${ }^{\circ}$ HighFidelity PCR Master Mix (New England Biolabs), $0.2 \mu \mathrm{M}$ of forward and reverse primers, and $10 \mathrm{ng}$ template DNA. Thermal cycling consisted of initial denaturation at $98^{\circ} \mathrm{C}$ for $1 \mathrm{~min}$, followed by 30 cycles of denaturation at $98{ }^{\circ} \mathrm{C}$ for $10 \mathrm{~s}$, annealing at $50^{\circ} \mathrm{C}$ for $30 \mathrm{~s}$, and elongation at $72{ }^{\circ} \mathrm{C}$ for $30 \mathrm{~s}$, finally $72^{\circ} \mathrm{C}$ for $5 \mathrm{~min}$. Same volume of $1 \times$ loading buffer (contained SYB green) was mixed with PCR products and operated electrophoresis on $2 \%$ agarose gel for detection. Then, PCR products were purified with GeneJET TM Gel Extraction Kit (Thermo Scientific, USA). Sequencing libraries were generated using Ion Plus Fragment Library Kit 48 rxns (Thermo Scientific, USA) following manufacturer's recommendations. The library quality was assessed on the Qubit@ 2.0 Fluorometer (Thermo Scientific, USA). At last, the library was sequenced on an Ion S5 TM XL platform to generate $400-600 \mathrm{bp}$ single-end reads. The library was sequenced on an Illumina HiSeq 2500 platform by Beijing Nuo He Zhi Yuan Technology Co., Ltd., China. 
Fecal SCFAs quantification by gas chromatography-mass spectrometer (GC-MS)

Quantification analysis of fecal SCFAs was performed using an Agilent 7890A gas chromatography coupled with an Agilent 5975C mass spectrometric detector (Agilent Technologies, USA) equipped with an HP-5MS column $(0.25 \times 30 \mathrm{~mm}, 0.25 \mu \mathrm{m}$ particlesize) (Suzhou Bionovogene Co., Ltd) as described previously [21]. Helium was used as a carrier gas at a constant flow rate of $1 \mathrm{~mL} / \mathrm{min}$. The initial oven temperature was held at $60{ }^{\circ} \mathrm{C}$ for $5 \mathrm{~min}$, ramped to $250^{\circ} \mathrm{C}$ at a rate of $10^{\circ} \mathrm{C} / \mathrm{min}$, and finally held at this temperature for $5 \mathrm{~min}$. The temperatures of the front inlet, transfer line and electron impact (EI) ion source were set as 280,250 and $230{ }^{\circ} \mathrm{C}$, respectively. Data handing was performed with an Agilent's MSD ChemStation (E.02.00.493, Agilent Technologies, Inc., USA).

\section{Statistical analysis}

The data are expressed as the mean \pm SEM. When the data were Gaussian distribution and variances were equal, differences among the groups were analyzed using one-way ANOVA followed by Tukey's post hoc test. Otherwise, the Kruskal-Wallis test and followed Dunn's post hoctesting were applied. Spearman's correlation analysis was performed to identify the correlations between microbiota and inflammatory indicators. The statistically significance level was considered as $P<0.05$ (Prism version 5.0 for Windows; GraphPad Software, San Diego, CA, USA).

\section{Results}

\section{Routine parameters of rats in diverse groups}

Body mass (BM)

There was no significant difference in initial BM among diverse groups. We found that $\mathrm{BM}$ in $\mathrm{DM} / \mathrm{CO}$ group were significantly decreased compared to $\mathrm{PF} / \mathrm{CO}$ group at week $1(P<0.001$, Fig. 1b). However, after 5 weeks of administration, $\mathrm{BM}$ in DM/FO group were notably increased compared with that in DM/CO group $(P<0.05$, Fig. 1b). These results demonstrated that dietary FO can maintain the BM in T2DM.

\section{Fasting blood glucose (FBG) and Glycated hemoglobin (GHb)}

FBG levels were significantly increased in diabetic rats compared to control rats (Fig. 1c). Intriguingly, the levels of FBG in DM/FO group were dramatically decreased compared with that in DM/CO group at week 4 and 5 $(P<0.05$, Fig. 1c). There was no significant difference of FBG levels between $\mathrm{PF} / \mathrm{CO}$ group and $\mathrm{PF} / \mathrm{FO}$ group (Fig. 1c). Similarly, $\mathrm{GHb}$ in $\mathrm{DM} / \mathrm{CO}$ group was higher than that in PF/CO group. However, the levels of $\mathrm{GHb}$ in $\mathrm{DM} / \mathrm{FO}$ group were lower than that in $\mathrm{DM} / \mathrm{CO}$ group $(P<0.05$, Fig. 1d).
Insulin (INS) and homeostasis model assessment of insulin resistance (HOMA-IR)

In addition, plasma INS $(P<0.001$, Fig. 1e) level were notably decrease in $\mathrm{DM} / \mathrm{CO}$ group compared to $\mathrm{PF} / \mathrm{CO}$ group. In DM/CO group, HOMA-IR $(P<0.001$, Fig. 1f) significantly increase compared to $\mathrm{PF} / \mathrm{CO}$ group. But INS and HOMA-IR remained no significant difference $(P>0.05)$ between DM/CO group and DM/FO group after the administration of dietary FO.

\section{Plasma lipid levels}

To assess the effects of dietary FO on lipid metabolism in rats, the plasma triglyceride (TG), total cholesterol (TC), low density lipoprotein cholesterol (LDL-C) and high density lipoprotein cholesterol (HDL-C) levels were investigated (Fig. 1g-j). The results showed that concentrations of plasma TC (Fig. 1h), TG (Fig. 1g) and LDL (Fig. 1i) in DM/FO group were effectively decreased, whereas HDL levels were increased, compared to DM/ CO group $(P<0.001$, Fig. 1 j).

\section{Serum SOD activity and MDA activity}

To investigate the effects of dietary FO on oxidative stress in diabetic rats, we examined the levels of serum SOD and MDA (Fig. 2). In DM/CO group, SOD level in serum significantly reduced compared to $\mathrm{PF} / \mathrm{CO}$ group. Feeding of diabetic rats with FO significantly increased SOD levels compared to the diabetic control group $(P<0.05$, Fig. 2a). However, serum MDA was significantly decreased in the supplementation with dietary FO in diabetes $(P<0.05$, Fig. $2 \mathrm{~b})$.

\section{Dietary FO reduced the plasma LPS levels}

Plasma levels of LPS in DM/FO group were significantly decreased compared to DM/CO group $(P<0.05)$, but still higher than that in $\mathrm{PF} / \mathrm{CO}$ group or $\mathrm{PF} / \mathrm{FO}$ group (Fig. 3), demonstrating that dietary FO possessed the ability to attenuate LPS generation from gram-negative pathogenic bacteria.

\section{Dietary FO altered plasma inflammatory cytokine levels in T2DM}

In attempt to investigate the effects of dietary FO on inflammation in diabetic rats, the levels of inflammatory cytokines including TNF- $\alpha$, IL-1 $\beta$, IL-6, IL-17A and IL10 were measured, respectively (Fig. $4 \mathrm{a}-\mathrm{e}$ ). Our results showed that the levels of IL-1 $\beta$ (Fig. 4a), TNF- $\alpha$ (Fig. 4b), IL-6 (Fig. 4c) and IL-17A (Fig. 4d) in DM/CO group were significantly increased compared to $\mathrm{PF} / \mathrm{CO}$ group, respectively. However, dietary FO administration remarkably decreased the contents of IL-1 $\beta(P<0.05)$ and TNF- $\alpha(P<0.05)$ in plasma, compared with that in DM/ CO group. Similarly, plasma IL-6 $(P<0.05)$ and IL-17A $(P<0.05)$ levels in DM/FO were also significantly reduced 


\section{A}

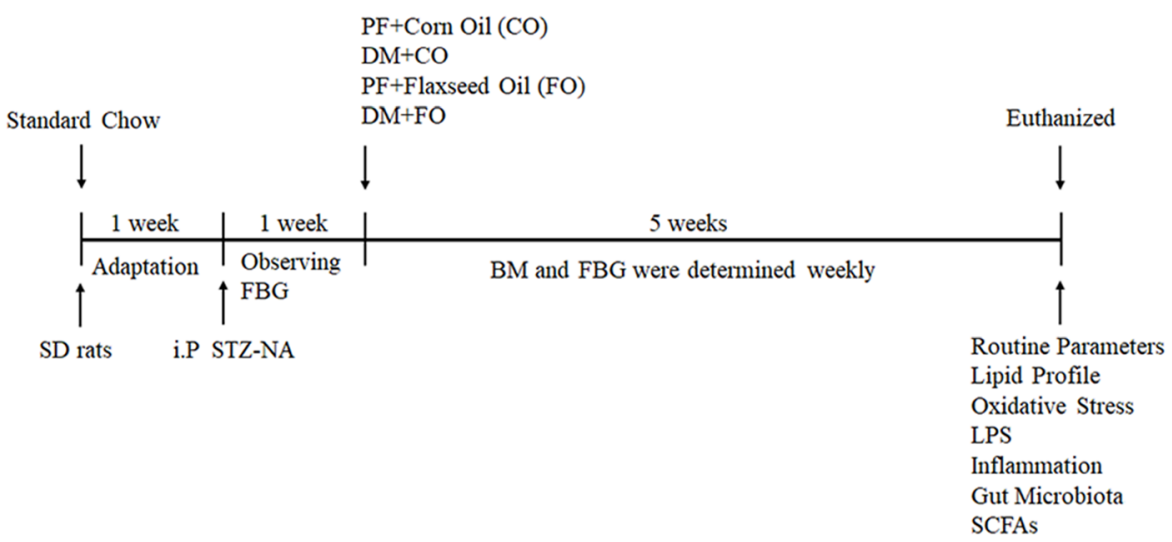

B

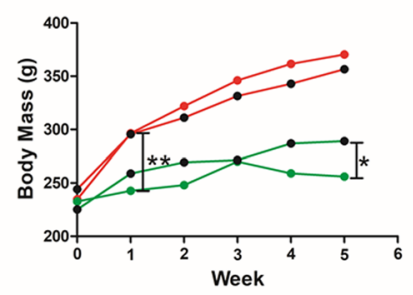

E

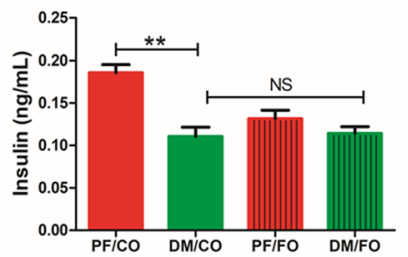

$\mathrm{H}$ TC

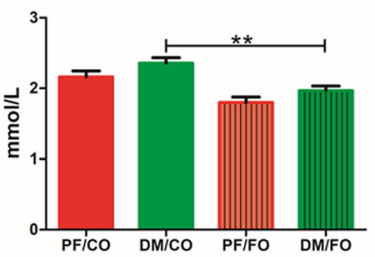

C
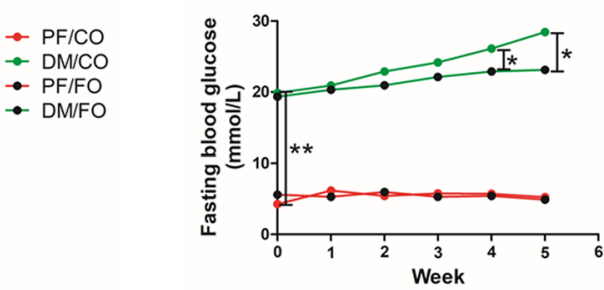

F

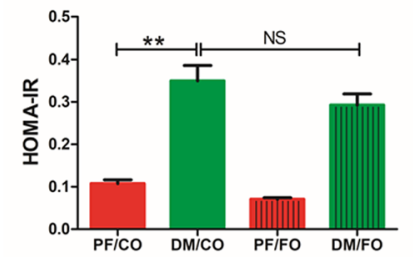

I

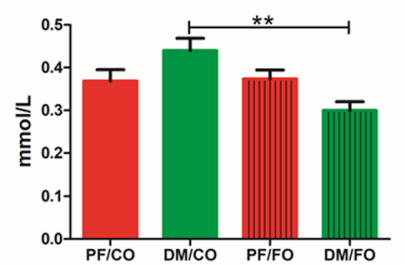

$\mathrm{D}$
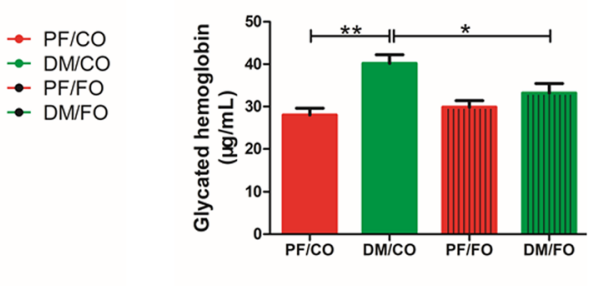

G

TG

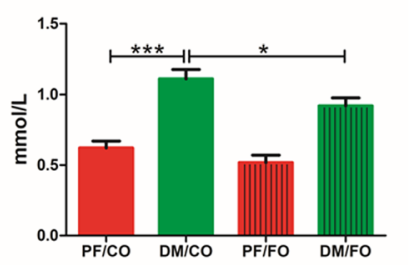

$\mathrm{J}$

HDL-C

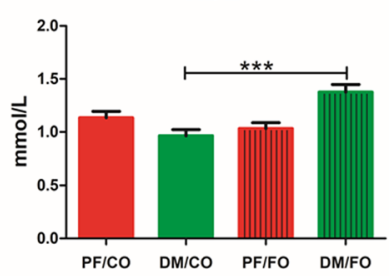

Fig. 1 Effects of dietary flaxseed oil treatment on glucolipid metabolism in diverse groups. a Schematic diagram of the study. SD rats with 200-250 g were conditioned for 1 week. Then T2DM model (FBG > $13.9 \mathrm{mmol} / \mathrm{L}$ ) were successfully induced by the intraperitoneal (i.p) administration of STZ-NA within another 1 week. For a subsequent period of 5 weeks, rats in diverse groups were respectively administrated with corn oil (CO) and flaxseed oil (FO) in control group (PF) or T2DM model group (DM). Body mass (BM) and FBG for each group were determined weekly. After 5 weeks of intervention, rats were euthanized and associated indications were investigated. $\mathbf{b}$ Body mass (BM); c Fasting blood glucose (FBG); d Glycated hemoglobin (GHb); e Insulin (INS); f Homeostasis model assessment of insulin resistance (HOMA-IR); $\mathbf{g}$ TG: Triglyceride; $\mathbf{h}$ TC: Total cholesterol; i LDL-C: Low density lipoprotein cholesterol; j HDL-C: High density lipoprotein cholesterol. Data was presented as mean \pm SEM $(n=6)$. Data was ${ }^{*} P<0.05,{ }^{* *} P<0.001$, NS not significant (one-way ANOVA followed by Tukey's post hoc test)

in comparison with those cytokines in DM/CO group. It showed no significant difference in plasma IL-10 levels between DM/CO group and DM/FO group ( $P>0.05$, Fig. $4 \mathrm{e})$. There results indicated that dietary FO treatment ameliorated the level of inflammation in rats with T2DM.

\section{Correlation analysis of LPS and inflammatory cytokines}

We analyzed the correlations among LPS and a series of inflammatory cytokines. LPS was positively correlated with IL-1 $\beta$, TNF- $\alpha$, IL- 6 and IL-17A (Additional file 3: Figure S2). 
A

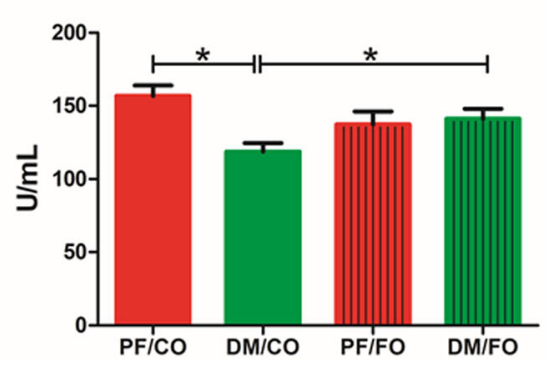

B

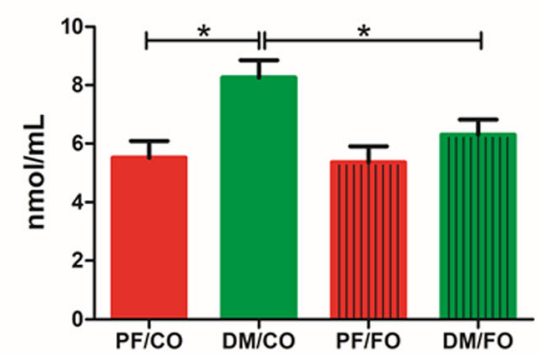

Fig. 2 Serum oxidative marker levels in the four groups of rats. a superoxide dismutase (SOD); b malondialdehyde (MDA). * $P<0.05$

\section{Dietary FO modulated gut microbiota in T2DM}

Accumulating studies have been addressing the close linkage between the changes to gut microbiota structure and T2DM $[3,5,22]$. To assess whether FO administration was associated with modulation of gut microbiota composition in diabetic rats, the fecal samples were analyzed by $16 \mathrm{~S}$ rRNA high throughput sequencing. Alpha-diversity was used to analyze the abundance and diversity of bacterial community, which was assessed by observed-species index and rarefaction curve. As shown in Fig. 5a, observed-species index analysis showed that the abundance and diversity of gut microbiota in DM/ $\mathrm{CO}$ group were the lowest in four groups, but the abundance and diversity of gut microbiota were altered after dietary FO treatment. However, the difference showed no significance $(p>0.05)$ by Tukey's post hoc test and Wilcoxon rank sum test. Rarefaction curves which were adopted to evaluate the rationality of the sequencing data (Fig. 5b), tended to be flat when the number of sequence increased to 10,000 , showing that the amount of sequencing data were reasonable. Beta-diversity for analyzing the overall composition of bacterial community was assessed by principal coordinates analysis (PCoA) (Fig. 6) and nonmetric multidimensional scaling (NMDS). PCoA results showed a significant difference of species in

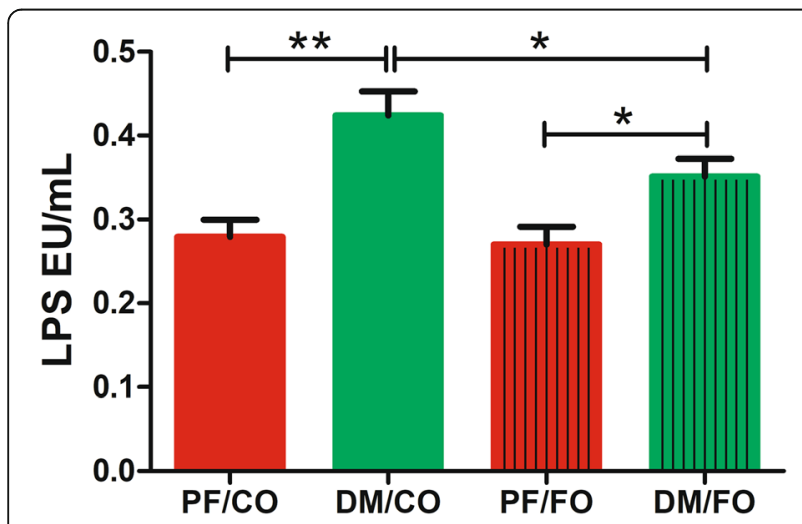

Fig. 3 Effects of dietary flaxseed oil treatment on plasma lipopolysaccharide (LPS) levels in T2DM. * $P<0.05$, ${ }^{* *} P<0.001$ fecal samples among the PF/CO group and DM/FO group compared to DM/CO group respectively (Fig. 7), indicating that dietary FO can alter gut microbiota of diabetic rats. In addition, NMDS analysis owned similar results (Additional file 4: Figure S3).

As shown above, to further explore the diversity of alpha-diversity and beta-diversity in composition of gut microbiota, the species distribution of gut microbiota from $\mathrm{PF} / \mathrm{CO}, \mathrm{DM} / \mathrm{CO}$ and $\mathrm{DM} / \mathrm{FO}$ groups were compared by operational taxonomic unit (OUT) analysis. As shown in venn diagram (Fig. 7), we found that there were 396 species shared among $\mathrm{PF} / \mathrm{CO}, \mathrm{DM} / \mathrm{CO}$ and $\mathrm{DM} / \mathrm{FO}$ groups. Intriguingly, 108 species were found in $\mathrm{PF} / \mathrm{CO}$ group, 46 species in DM/CO group and 40 OTUs in DM/FO group.

After 5 weeks of FO intervention, at phylum level, we found that Firmicutes and Bacteroidetes constituted two dominant phyla in each group (Fig. 8a). The proportion of Firmicutes was significantly elevated in DM/CO group compared to PF/CO group, which was reversed by dietary FO (Fig. 8b). The relative abundance of Bacteroidetes was significantly reduced in $\mathrm{DM} / \mathrm{CO}$ group than that in $\mathrm{PF} /$ CO group $(\mathrm{P}<0.05$, Fig. $8 \mathrm{c})$ or $\mathrm{DM} / \mathrm{FO}$ group $(P<0.05$, Fig. 8c). In addition, our results also indicated that DM rats had a higher ratio of Firmicutes-Bacteroidetes, whereas FO intervention reduced the abnormal ratio $(P<0.05$, Fig. $8 d)$. Taken together, our data revealed that under experimental conditions, FO supplementation had a major effect on Firmicutes and Bacteroidetes.

To further understand the composition of gut microbiota in T2DM, we analyzed top 40 taxa of genus level in diverse groups (Fig. 9). We found that the proportion of Blautia in DM/CO group was higher than that in PF/ $\mathrm{CO}$ group, whereas FO intervention obviously reduced the relative abundance $(P<0.05$, Fig. 9b). In contrast, the proportion of Alistipes was decreased in DM/CO group, but FO treatment sharply elevated the relative abundance of Alistipes in diabetic rats $(P<0.05$, Fig. 9c). Collectively, these results indicated that dietary FO obviously altered the initial proportion of OTUs at genus level, mainly including Blautia and Alistipes. 


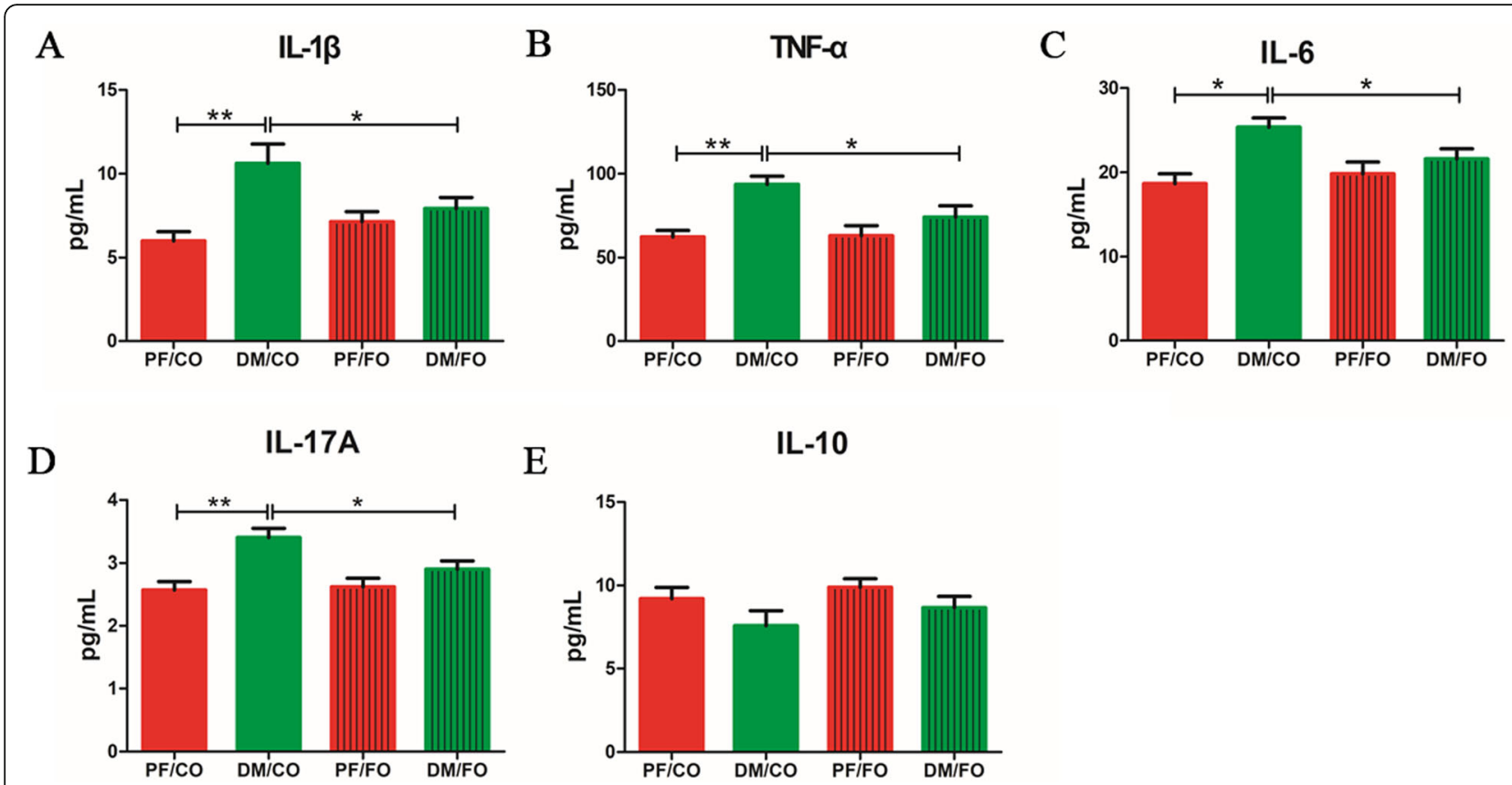

Fig. 4 Plasma of rats from diverse groups were collected respectively for detection of IL-1ß (a), TNF-a (b), IL-6 (c) and IL-17A (d) and IL-10 (e) concentrations using ELISA kit. * $P<0.05,{ }^{* *} P<0.001$

\section{Gut microbiota was correlated with inflammatory indicators}

Correlations between a series of inflammatory indicators and the proportions of the above differential bacteria were analyzed at phylum and genus levels, respectively (Fig. 10). Our results showed that the relative abundance of Firmicutes was positively correlated with the levels of IL-1 $\beta(P=0.0035$, Fig. 10a), TNF- $\alpha(P=0.0475$, Fig. 10b $)$, IL-6 $(P=0.0038$, Fig. 10c), IL-17A $(P=0.0191$, Fig. $10 \mathrm{~d})$ and LPS $(P=0.0496$, Fig. 10e), respectively. Moreover, Bacteroidetes abundance was negatively correlated with IL-1 $\beta$ ( $P=0.0006$, Fig. 10h $)$ TNF- $\alpha(P=0.0022$, Fig. 10f $)$, IL-17A $(P=0.0031$, Fig. $10 \mathrm{~g})$ and LPS $(P=0.0028$, Fig. 10i), respectively. At genus level, the Blautia abundance showed a positive association with IL-1 $\beta$ ( $P=0.0014$, Fig. $10 \mathrm{j})$, TNF- $\alpha(P=0.0041$, Fig. $10 \mathrm{k})$, IL-6 $(P=0.0005$, Fig. 10l) and LPS $(P=0.0069$, Fig. $10 \mathrm{~m})$. Alistipes abundance was negatively correlated with TNF-

\section{A}

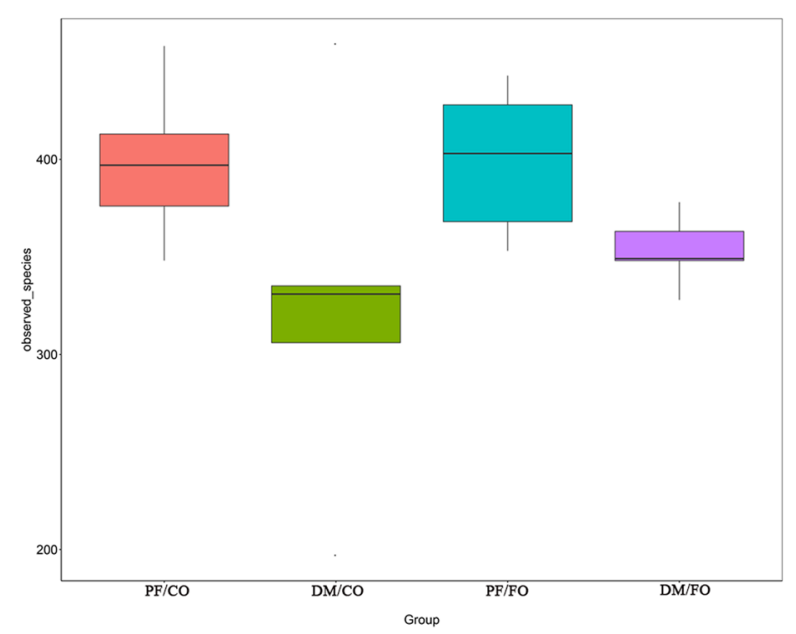

B

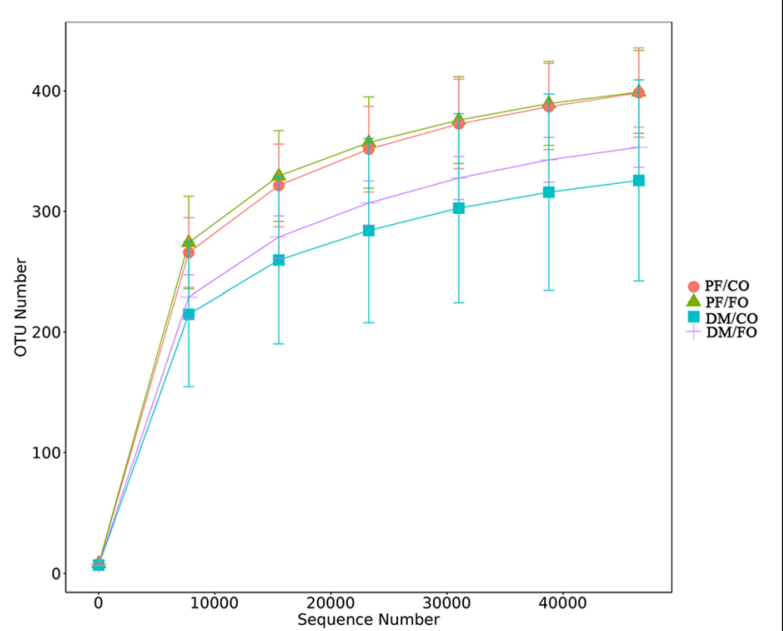

Fig. 5 Alpha-diversity analysis showing difference in terms of abundance and diversity of gut microbiota in diverse groups. a Observed-species index; $\mathbf{b}$ Rarefaction curve 


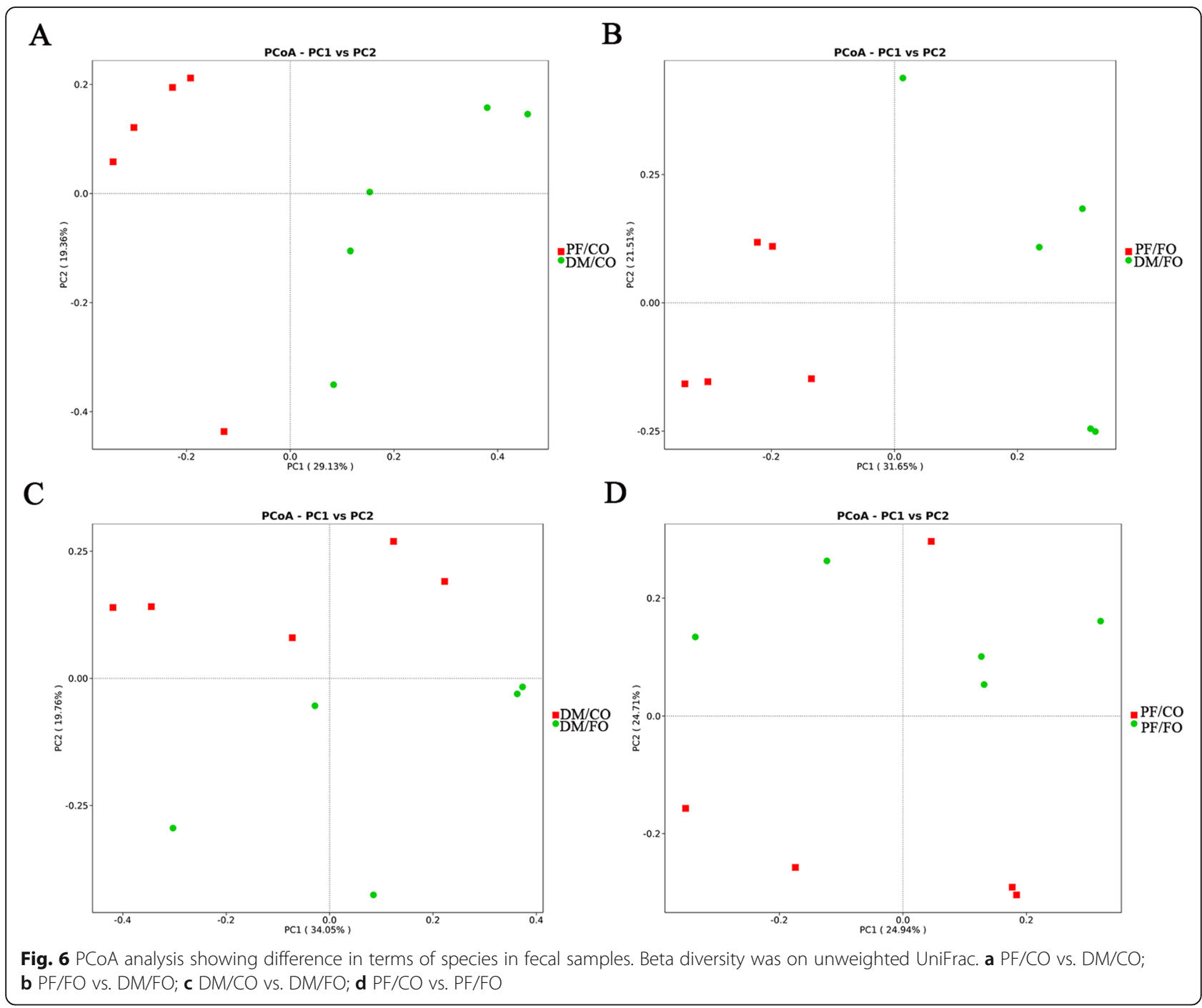

$\alpha(P=0.0088$, Fig. 10n $)$ and LPS $(P=0.0478$, Fig. 10o $)$. These results indicated that gut microbiota and inflammatory indicators interfered and closely correlated.

\section{FO increased the SCFAs in fecal intestinal metabolites}

The fecal concentrations of SCFAs mainly including acetic acid, propionic acid and butyric acid, were determined by GC-MS. The results showed that the amount of acetic acid $(P=0.046$, Fig. 11b), propionic acid $(P=$ 0.149 , Fig. 11c) and butyric acid $(P=0.201$, Fig. 11d) of the DM/CO group was decreased compared with that of the PF/CO group. However, the fecal concentration of acetic acid $(P<0.001$, Fig. $11 \mathrm{~B})$, propionic acid $(P<0.001$, Fig. 11c) and butyric acid $(P<0.05$, Fig. 11d) in DM/FO group was significantly increased compared with that of the DM/CO group. As shown in Fig. 12, we also found that SCFAs (acetic acid, propionic acid and butyric acid) was negatively correlated with inflammatory cytokines. These results indicated that FO promotes SCFAs production after increasing the abundance of beneficial bacteria.

\section{Discussion}

In this study, we investigated the therapeutic effects and associated mechanisms of dietary FO administration on T2DM induced by STZ and NA in rats. Intraperitoneal injection of STZ is mainly attributed to overproduction of reactive oxygen species (ROS) leading to toxicity of pancreatic cells [23], which regulate the synthesis and release of insulin via the glucose transporter (GLUT) and alkylation of DNA. In contrast, NA prevents excess damage to pancreatic $\beta$ cells during the induction of T2DM. This T2DM rat model has been widely used to assess the effects and mechanisms of intervention [19, 24-26].

Our results demonstrated that supplementary FO showed more effective in alleviation of T2DM, which may be due to the suppression of inflammation and restoration of gut dysbiosis, potentially providing a theoretical 


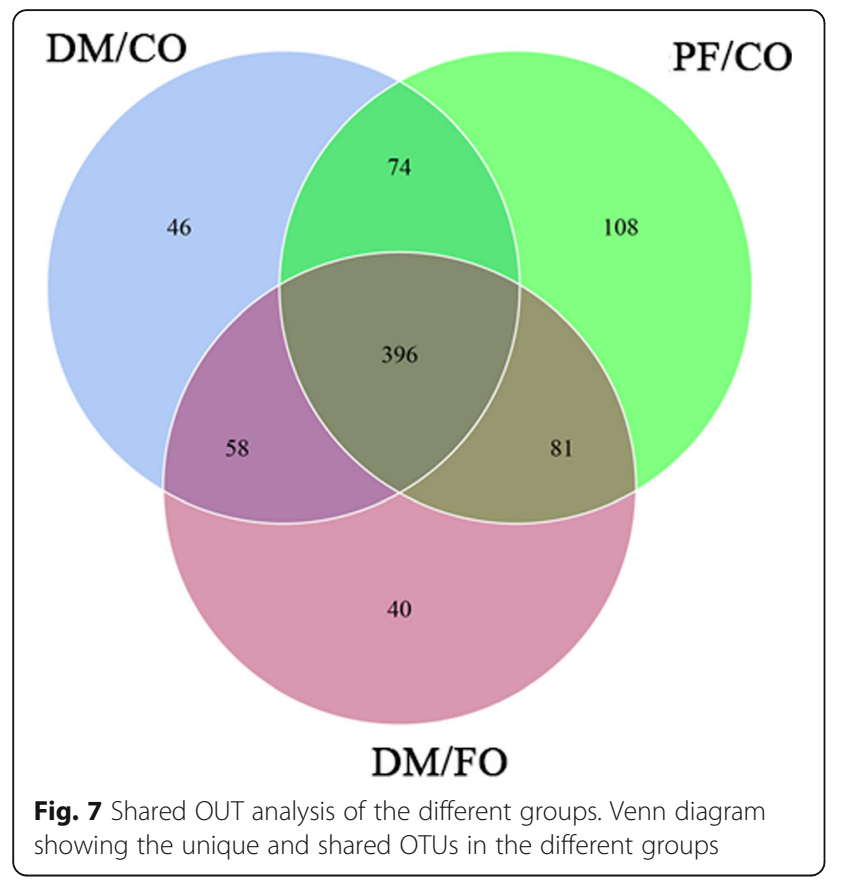

foundation as an inexpensive intervention for prevention and treatment of T2DM.

Consumption of $\omega-3$ PUFAs are beneficial for chronic metabolic diseases including T2DM via anti-inflammation and suppressing oxidative stress in clinical and experimental studies $[12,13]$. FO, as a source of a plant-derived $\omega-3$ PUFAs (rich in ALA), has been indicated to show antiinflammatory properties extensively investigated on the whole body [12, 13]. Wang F et al [27] showed that Perilla oil (rich in ALA) supplementation improved hypertriglyceridemia and gut dysbiosis in diabetic KKAy mice. A randomized double-blind placebo-controlled clinical trial revealed that FO supplementation significantly improved levels of gene expression related to insulin, lipid and inflammation in diabetic patients with coronary heart disease [28]. Our results demonstrated that BM were lower in $\mathrm{DM} / \mathrm{CO}$ group, but elevated after FO intervention in the STZ-NA induced diabetic rat model, which was consistent with previous study [8], indicating that FO may positively affect nutrients absorption and efficiency of calorie utilization in gastrointestinal tract in T2DM. Yang, $\mathrm{Z}$. H et al [29] found that an increase in $\omega-3$ PUFA levels and the concomitant decrease in the $\omega-6 / \omega-3$ PUFA level

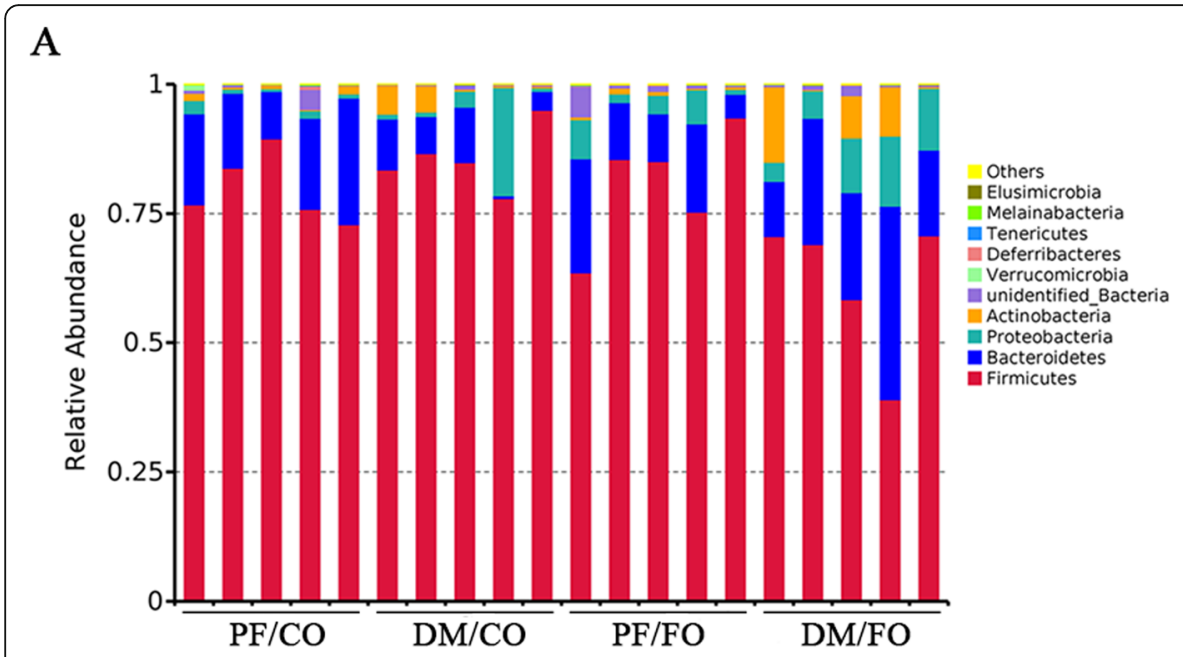

B

Firmicutes

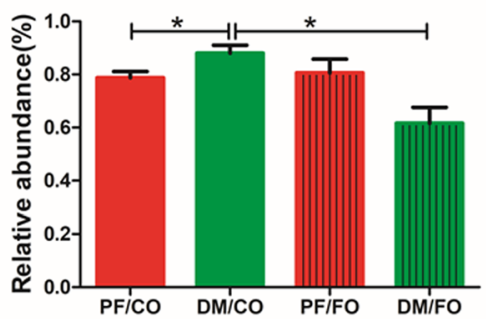

C

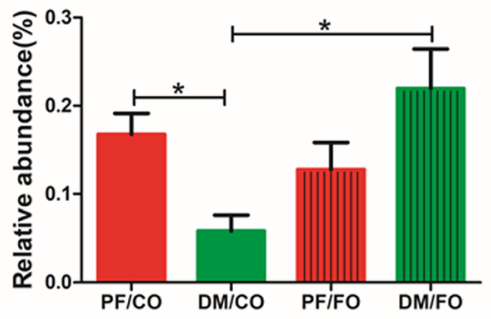

$\mathrm{D}$

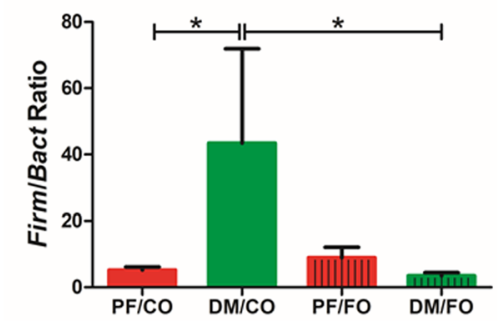

Fig. 8 Relative abundance of microbial species at the phylum level in diverse groups of rat feces. a The relative abundance of microbial species at the phylum level in the feces of rats; $\mathbf{b}$ Firmicutes; $\mathbf{c}$ Bacteroidetes; $\mathbf{d}$ the ratio of Bacteroidetes-Firmicutes. ${ }^{*} P<0.05$ 


\section{A}

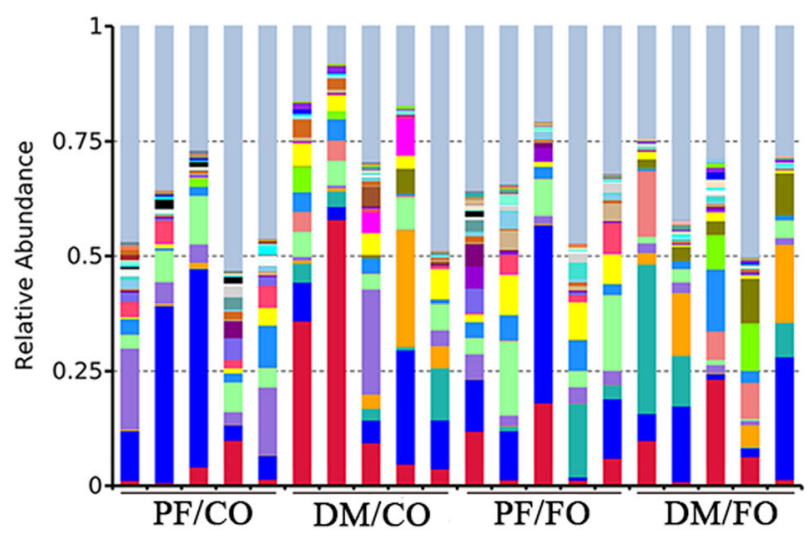

\begin{tabular}{|c|c|c|}
\hline $\begin{array}{l}\text { robacter } \\
\text { la } \\
\text { illa } \\
\text { ides } \\
\text { cterium }\end{array}$ & $\begin{array}{l}\text { des } \\
\text { tia } \\
\text { a } \\
\text { Is }\end{array}$ & $\begin{array}{l}\text { bacter } \\
\text { occus } \\
\text { tobacter } \\
\text { ostipes } \\
\text { inimonas } \\
\text { mansia } \\
\text { coccus } \\
\text { vibrio } \\
\text { onas } \\
\text { creutzia }\end{array}$ \\
\hline
\end{tabular}

B

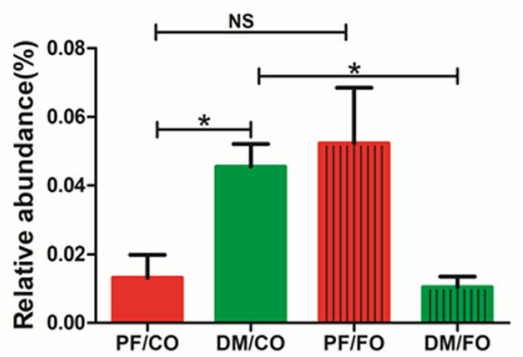

C

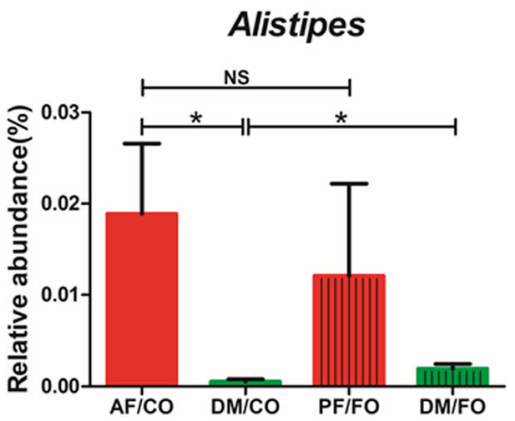

Fig. 9 Relative abundance of microbial species at the genus level in the feces. a The relative abundance of microbial species at the genus level in the feces of rats; b Blautia; c Alistipes; * $P<0.05$, NS not significant

ratio were likely to be involved in the beneficial changes to the metabolic indicators. Interestingly, we also found that FO treatment effectively alleviated routine parameters including FBG, GHb, liver HE (Additional file 5: Figure S4) and metabolic indicators (TC, TG, LDL and HDL), which revealed that $\mathrm{FO}$ can regulate the metabolic disorders in consistent with a recent studies [8].

Oxidative stress is considered to be one of the chief factors responsible for the development and progression of T2DM. Haliga, R et al [30] showed that dietary flaxseed reduced renal oxidative stress by improving activity of SOD and decreasing thiobarbituric acid reacting substances (TBARS) in STZ-induced diabetic hamster model. In this study, dietary FO induced an increase of serum oxidative indicator SOD as well as a decrease of MDA, which was paralleled with previous study [31]. Collectively, it is indicated that oxidative stress may be linked to the etiology of T2DM and ameliorated by a healthy diet.

LPS derived from pathogenic bacteria, a causal link between gut microbiota and systemic low-grade inflammation, translocates to liver and binds to TLR-4 to induce inflammatory cascade reaction to ultimately lead to diabetes mellitus [32]. Our data showed that plasma LPS was notably decreased after FO intervention, indirectly demonstrating that integrity and permeability of intestinal mucosa barriers were improved to alleviate peripheral endotoxemia. The damage of intestinal tight junction proteins like Zo-1 and Occludin is essential and critical for LPS translocation. However, the direct evidence of gut permeability and integrity still needs further research to measure and assess the efforts of the intestinal mucosal barrier, including physical tight junctions (Zo-1, Occludin) and other components of the gut barriers involving 
A

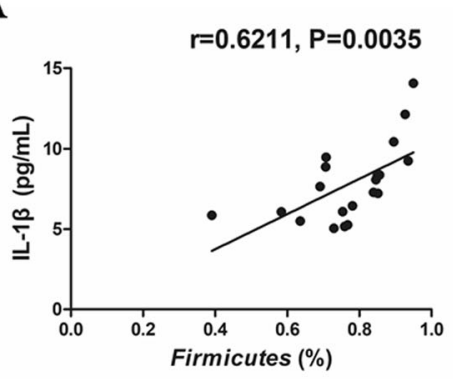

D

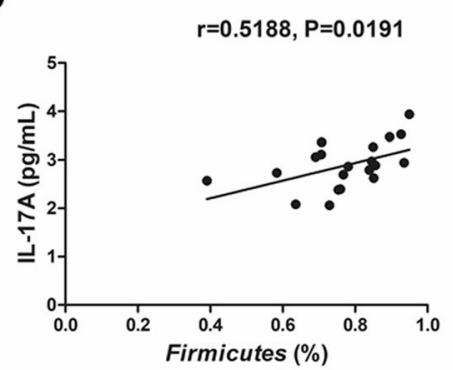

G

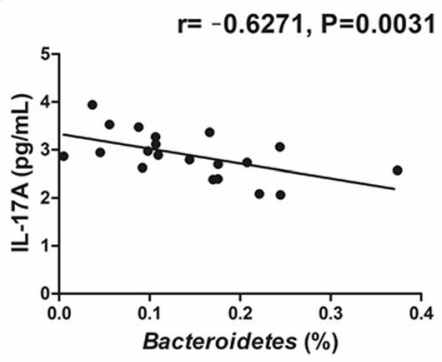

J

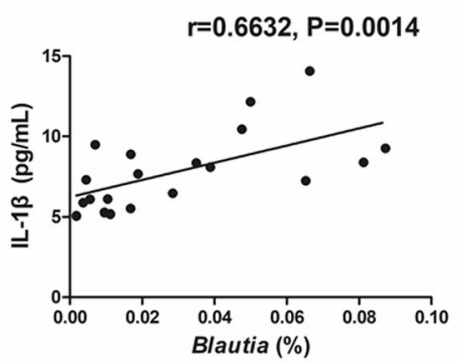

M

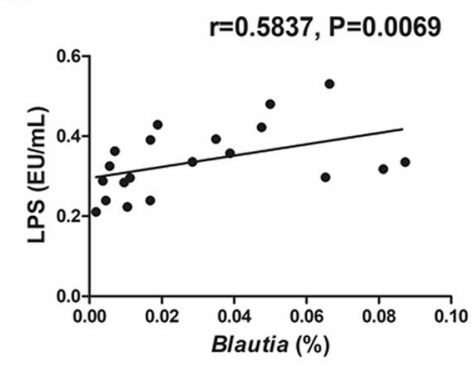

B

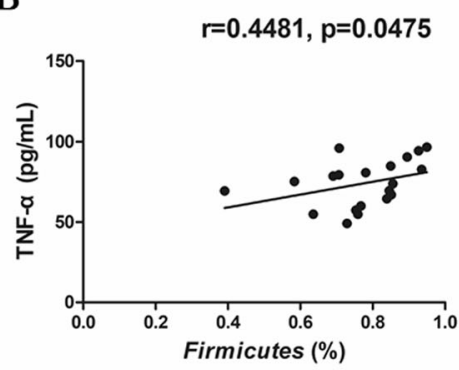

E

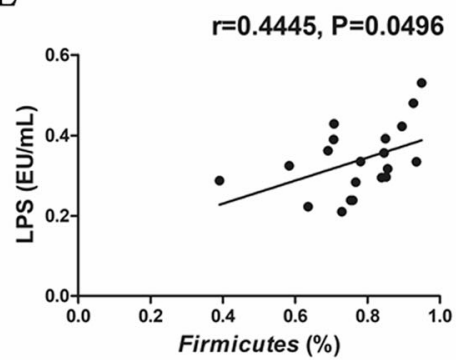

$\mathrm{H}$

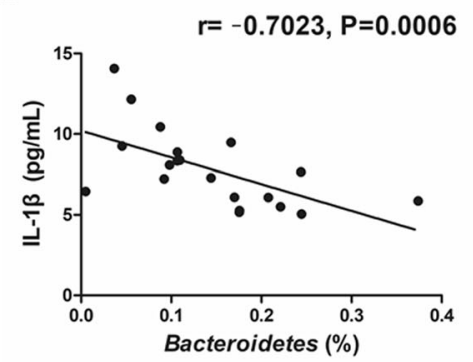

K

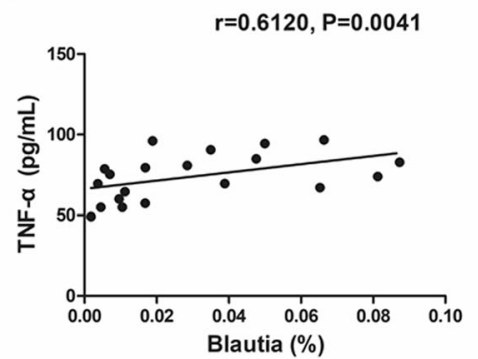

$\mathrm{N}$

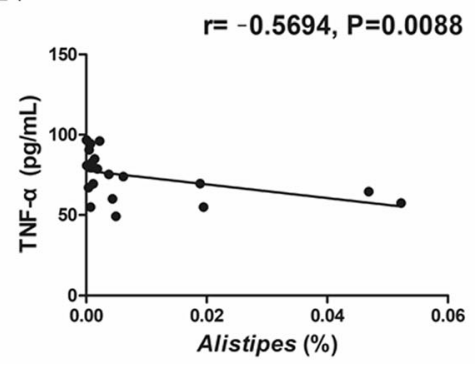

C

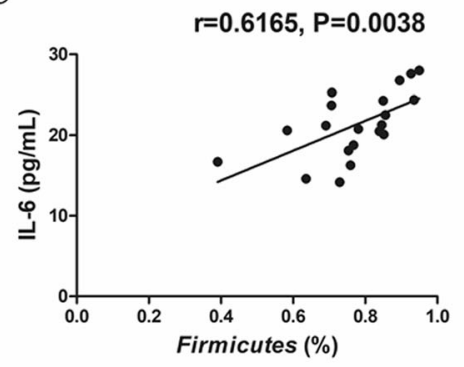

F

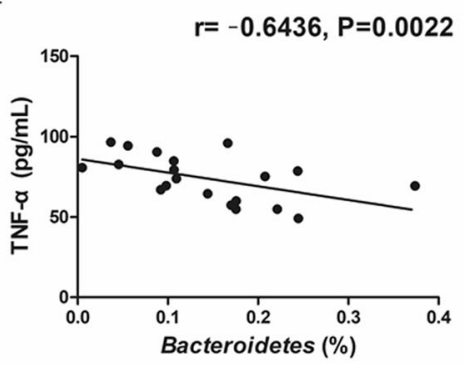

I

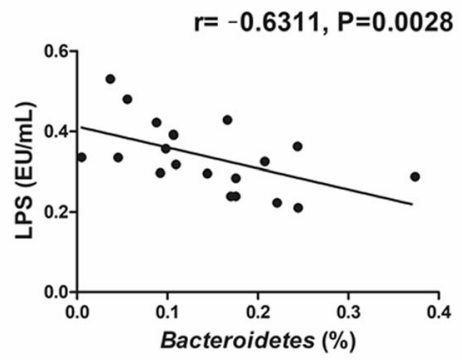

$\mathrm{L}$

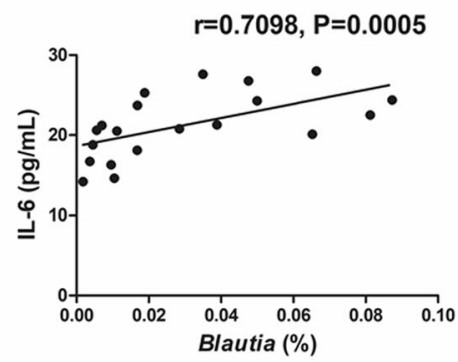

$\mathrm{O}$

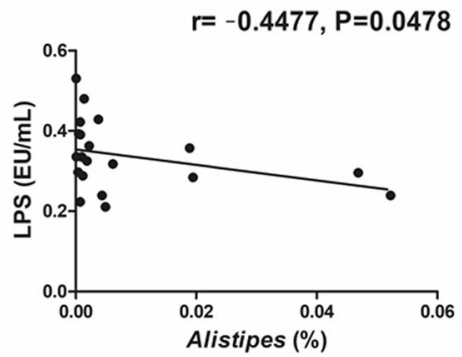

Fig. 10 Correlations between inflammatory cytokine and bacterial abundance at the phylum level or genus in the feces of rats. a-e Firmicutes

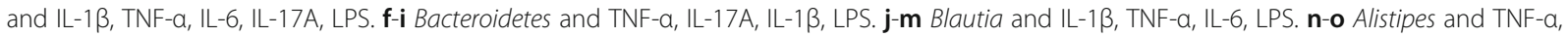
LPS, respectively 


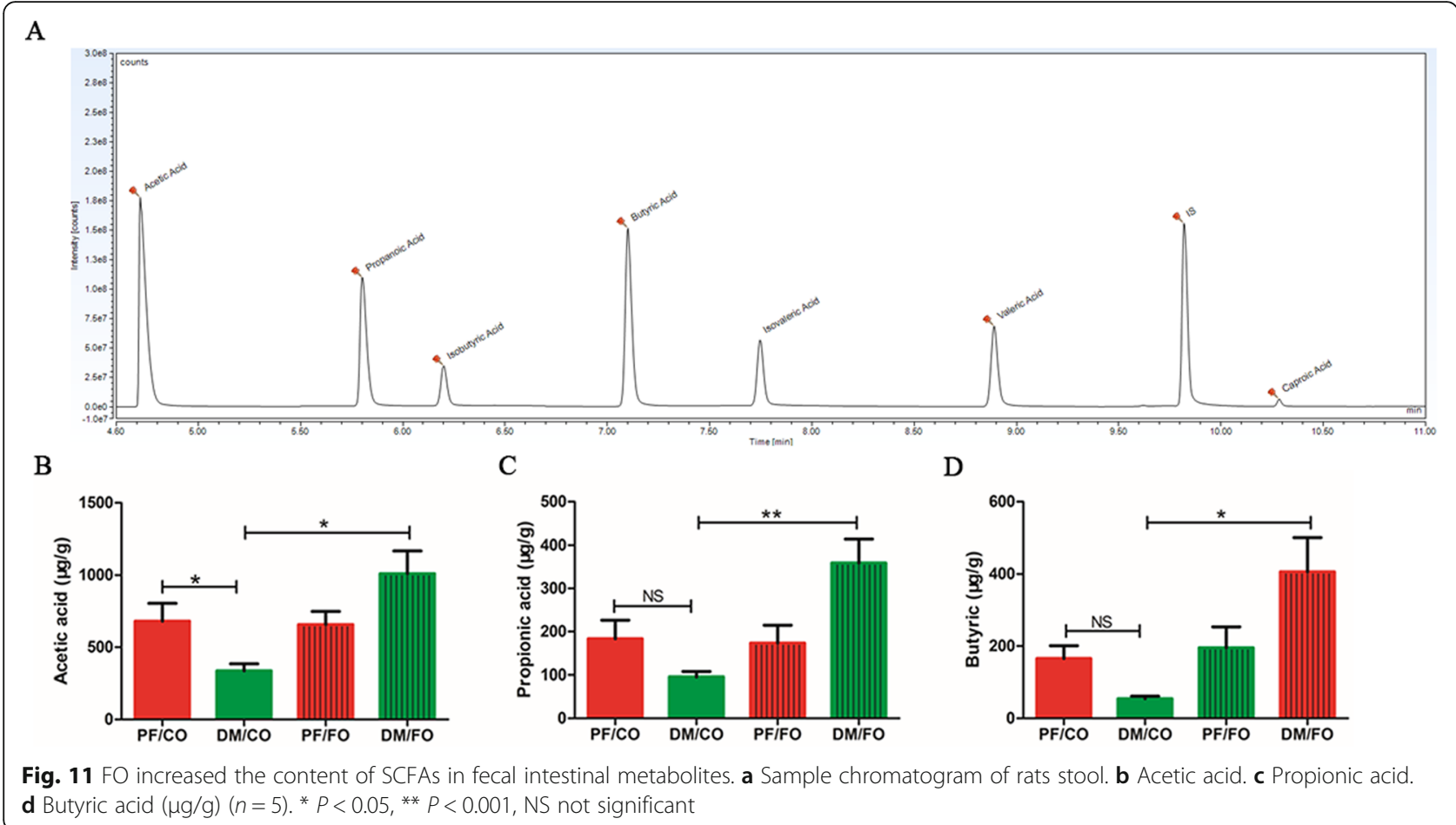

intestinal mucosal immunity $(\gamma \delta \mathrm{T}$ cells, intraepithelial lymphocytes (IELs), Tregs, natural killer T (NKT) cells).

Diabetes present a chronic metabolic disease with a low-grade inflammation [33]. Studies have demonstrated that activation of neutrophils and macrophage results to oxidative stress as well as inflammatory cytokines release including IL-1 $\beta$, IL- 6 and TNF- $\alpha$, leading to the decrease of insulin secretion, hepatic steatosis and a series of metabolic disorders in diabetes [34]. Hotamisligil, G et.al [35] firstly showed that the proinflammatory cytokine TNF- $\alpha$ was able to induce insulin resistance.

Accelerating studies have demonstrated that $\omega-3$ fatty acids are considered to possess anti-inflammatory ability in chronic metabolic diseases [13]. In T2DM, inflammation and glucose are interrelated, with reciprocal causation [36]. High glucose concentration leads to process of protein glycation and the production of advanced glycose end products (AGEs). As a consequence, the accumulation of AGEs activates NF- $\mathrm{kB}$ to induce TNF- $\alpha$ expression which lead to chronic inflammation [37]. Intakes of dietary $\omega-3$ fatty acids are associated with reduced lymphocyte proliferation and Th1 cell development [38], lower circulating levels of leptin, C-reactive protein and other proinflammatory cytokines, as well as a lower risk of infection [39]. It has been shown that supplementation of FO for 8 weeks caused a significant decrease in the levels of glucose tolerance, IL-1 $\beta$ and TNF- $\alpha$ in obese and diabetic [8]. Our results showed that a significant augmentation in a series of pro-inflammatory cytokines including IL- $1 \beta$, TNF- $\alpha$, IL-6 and IL-17A in STZ-NA induced diabetic rats.
However, the levels of those pro-inflammatory cytokines were dramatically reduced after FO treatment, which was in agreement with a recent study where dietary FO modulated expression of inflammatory genes with alleviation of protein glycation status in STZ-NA induced diabetic rats [40]. The possible mechanism of anti-inflammation is that ALA in FO diet-mediated GPR120 activation and $\beta$ arrestin 2 recruitment in the inhibition of TLR4 and TNF$\alpha$ downstream signaling. But the exact evidence needs to be further researched. IL-10 is an anti-inflammatory cytokine released by Kupffer cells and monocytes. But in present study, we found IL-10 showed no significant difference among diverse groups, which was not paralleled with previous study of T2DM [41]. We speculated that IL10 maybe play a complicated role in regulating pro-and anti-inflammation during T2DM. Additionally, regulatory immune cells especially regulatory $\mathrm{T}$ lymphocytes (Tregs), which may play a critical role in regulation of inflammation to keep maintain immune balance in T2DM. However, the roles of IL-10 and Treg cells in underlying mechanisms of FO-treated T2DM need to be further investigated.

Growing evidences have demonstrated the gut microbiota play a critical role in the development of T2DM [3, 5 , 27]. Gut dysbiosis can facilitate LPS entry into systemic circulation through increasing gut permeability, which leads to inflammation and metabolic dysfunction [42]. In this study, at phylum level, we found that Bacteriodetes and Firmicutes were the most dominant in all four groups, which was similar to the human intestinal 
A

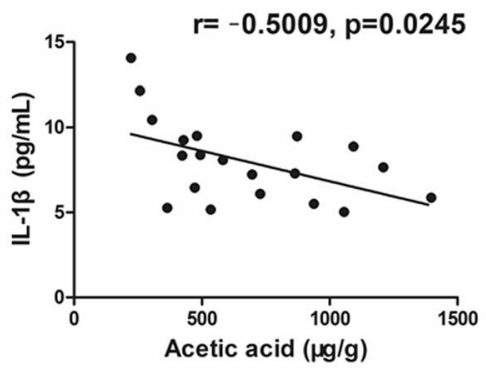

C

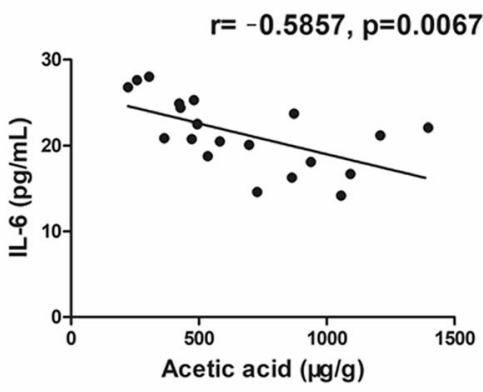

E

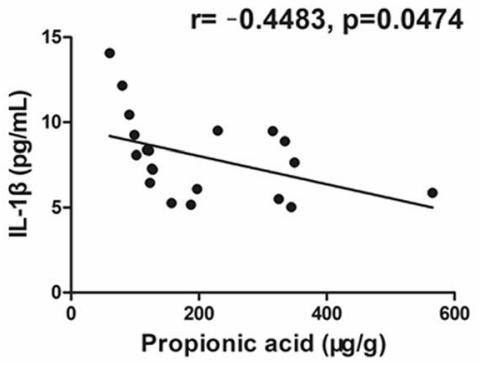

G

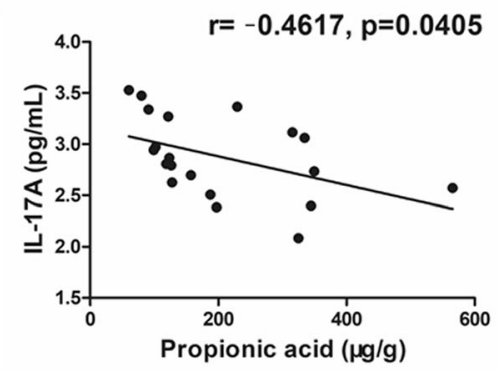

I

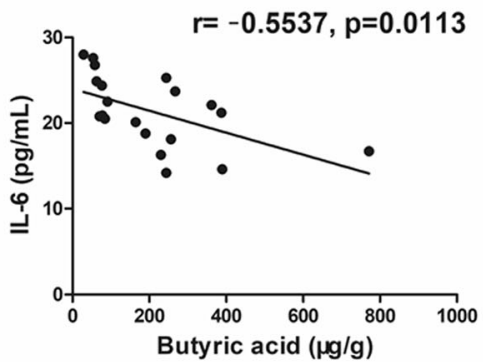

B

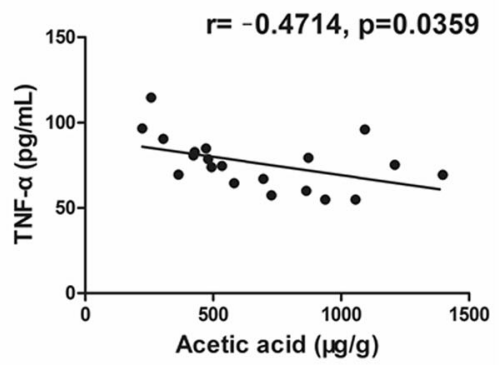

D

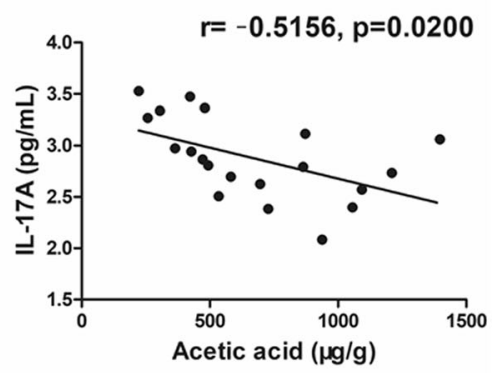

F

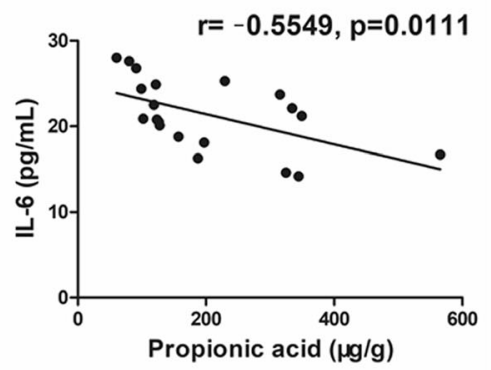

$\mathrm{H}$

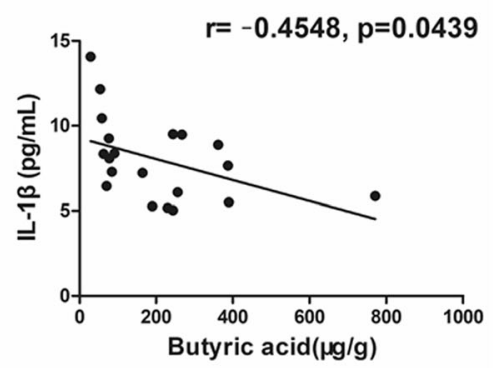

J

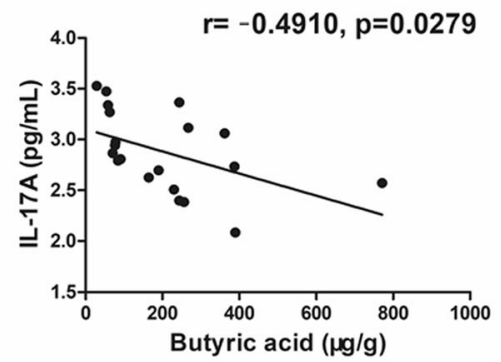

Fig. 12 Correlation analysis between SCFAs and liver inflammatory cytokines. a Acetic acid and IL-1 $\beta$; b Acetic acid and TNF-a; $\mathbf{c}$ Acetic acid and IL-6; d Acetic acid and IL-17A; e Propionic acid and IL-1ß; f Propionic acid and IL-6; g Propionic acid and IL-17A; h Butyric acid and IL-1B; i Butyric acid and IL-6; j Butyric acid and IL-17A 
flora [43]. Firmicutes played a major role in absorbing calories from the diet and storing fat in gut cells. In present study, a decrease of Firmicutes after FO treatment revealed that FO may attenuate diabetes via reducing Firmicutes-involved in energy absorption. In addition, the ratio of Firmicutes-Bacteroidetes was increased in diabetic group, suggesting a characteristics of micro-ecological disorder in intestinal microbes [5]. Importantly, dietary FO intervention restored this ratio, demonstrating that dietary FO positively shaping the host microbial ecosystem [44].

In our results, positive correlations of Firmicutes with pro-inflammatory IL-1 $\beta$, TNF- $\alpha$, IL-6, IL-17A and LPS demonstrated that both pro-inflammatory indicators and Firmicutes contributed to pathogenesis of diabetes. In contrast, negative correlations of Bacteroidetes with proinflammatory indicators may stand for a protection in diabetes. However, the details in relationship between Firmicutes-Bacteroidetes and inflammation need to be further investigated.

At the genus level, Blautia is a gram-positive, anaerobe bacterium belonging to the family Lachnospiraceae, which was thought to take part in the development of glucose metabolism disturbances [45]. A recent study also showed that Blautia, especially Blautiacoccoides, may activate secretion of TNF- $\alpha$ concentration with an even greater extent than the LPS [46]. In our study, we found that increased Blautia was positively correlated with inflammatory indicators (IL- $1 \beta$, TNF- $\alpha$, IL-6 and LPS) in diabetes. Meanwhile, we found that the abnormal elevated Blautia in diabetes was restored by FO intervention. Similarly, ALA rich in Perilla oil supplementation resulted in an decreased abundance of Blautia in diabetic KKAy mice [27]. In addition, we found that FO treatment significantly regulated the abundance of Alistipes which belongs to family Rikenellaceae. Consistently, Alistipes was decreased in gestational diabetes mellitus based on a recent study [47]. Furthermore, we found that Alistipes was negatively correlated with TNF- $\alpha$ and LPS, demonstrating that Alistipes may suppress diabetic inflammation. The above findings suggested that Alistipes may be involved in the improvement of diabetes.

More and more studies have suggested that SCFAs, one of critical gut microbiota metabolites, as a link between microbiota and host homeostasis, play an important role in regulation of inflammation, glucose and lipid metabolism. Acetic acid, propionic acid and butyric acid mainly account for $90-95 \%$ of the total SCFAs [48]. Acetate and propionate are the main products of Bacteroidetes and butyrate is mainly produced by Firmicutes [49]. A study showed that $\omega-3$ PUFA exerted a positive action by reverting microbiota-derived SCFAs [50]. Thus, in this study, restores of abnormal decreased acetic acid with FO administration revealed that dietary
FO may alleviate diabetes via modulating gut microbiota acetic acid. In addition, increased Bacteroidetes, decreased Firmicutes, and reduced ratio of Firmicutes-Bacteroidetes after dietary FO treatment demonstrated that Bacteroidetes acted the main source of increased acetate. Intriguingly, dietary FO supplementation also significantly increased propionic acid and butyric acid which showed decreased in diabetes group but without significant difference, suggesting that dietary FO possessed the ability to increase SCFAs within or without diabetes. But a study reported that oral butyrate administration significantly alleviated diabetic-endotoxemia with improved gut integrity and decreased ratio Firmicutes-Bacteroidetes [51]. The exact role of increased propionic acid and butyric acid after dietary FO intervention needs to be further investigated. The above suggested that dietary FO may regulate gut microbiota metabolites SCFAs, especially acetate with beneficial effects on diabetes.

\section{Conclusion}

This study highlighted that dietary FO supplementation ameliorated T2DM induced by STZ-NA via antiinflammation, modulating composition of gut microbiota and gut microbiota metabolite acetate in rat, suggesting that it can potentially serve as inexpensive interventions for the prevention and treatment of diabetes.

\section{Supplementary information}

Supplementary information accompanies this paper at https://doi.org/10. 1186/s12944-019-1167-4

Additional file 1: Table S1. Fatty acid composition (\%) of dietary fats contained.

Additional file 2: Figure S1. Size distribution was estimated by electrophoresis. (number 6-10is the size distribution in NC/CO group, number $11-15$ is the size distribution in NC/FO group, number 21-25 is the size distribution in DM/CO group and number 26-30 is the size distribution in DM/FO group). Correlation analysis of LPS and inflammatory cytokines. (A) IL-1 $\beta$ and LPS; (B) TNF-a and LPS; (C) IL-6 and LPS; (D) IL-17A and LPS.

Additional file 3: Figure S2. Correlation analysis of LPS and inflammatory cytokines. (A) IL-1 $\beta$ and LPS; (B) TNF-a and LPS; (C) IL-6 and LPS; (D) IL-17A and LPS. Effects of different dietary oil on liver injury and in T2DM. Representative images of hepatic hematoxylin and eosin (H\&E) staining. $\mathrm{CV}$, central vein; $\mathrm{NH}$, normal hepatocyte; $\mathrm{DH}$, degeneration of hepatocytes.

Additional file 4: Figure S3. NMDS analysis showing difference in terms of species in fecal samples. (A) PF/CO vs. DM/CO; (B) PF/FO vs. DM/ FO; (C) DM/CO vs. DM/FO; (D) PF/CO vs. PF/FO. Size distribution was estimated by electrophoresis. (number 6-10 is the size distribution in NC/ CO group, number 11-15 is the size distribution in NC/FO group, number $21-25$ is the size distribution in DM/CO group and number 26-30 is the size distribution in DM/FO group).

Additional file 5: Figure S4. Effects of different dietary oil on liver injury and in T2DM. Representative images of hepatic hematoxylin and eosin (H\&E) staining. $\mathrm{CV}$, central vein; $\mathrm{NH}$, normal hepatocyte; $\mathrm{DH}$, degeneration of hepatocytes. NMDS analysis showing difference in terms of species in fecal samples. (A) PF/CO vs. DM/CO; (B) PF/FO vs. DM/FO; (C) DM/CO vs. DM/FO; (D) PF/CO vs. PF/FO. 


\section{Abbreviations}

ALA: a-linolenic acid; CO: Corn oil; FO: Flaxseed oil; IL: Interleukin; INS: Insulin; LPS: Lipopolysaccharide; MDA: Malondialdehyde; OTUs: Operational taxonomic units; PUFAs: Polyunsaturated fatty acids; SCFAs: Short chain fatty acids; SOD: Superoxide dismutase; STZ-NA: Streptozotocin-nicotinamide; T2DM: Type 2 diabetes mellitus; TLR-4: Toll-like receptor-4; TNF-a: Tumor necrosis factor-a

\section{Acknowledgements}

Not applicable.

\section{Authors' contributions}

WH, ZXX, ZLL and SLP designed and wrote the paper. ZLL, LK, WZ, SLP, WT, LYW, LP, DXY, DYP, WH and ZXX performed research. All authors have read and approved the final manuscript.

\section{Funding}

This work was supported by the research and development plan of the 13th five-year plan of Ningxia autonomous region (the major S\&T projects), China (Grant No. 2016BZ02); The first class discipline construction project in Colleges and Universities of Ningxia, China (Grant No. NXYLXK2017A05); The first class discipline project in College of Traditional Chinese Medicine in Ningxia Medical University, China (NXYLXK2017A06); Ningxia High School first-class Disciplines (West China first-class Disciplines Basic Medical Sciences at Ningxia Medical University), China (Grant No. NXYLXK2017B07); The Nationa Key Research and Development Program of China (No. 2016YFD0400605); The Construction of Clinical Research Platform for Gut Microecology and Endocrine Diseases, China, the Research Project of Ningxia Medical University, China (No. XT2018007) and the research project of Ningxia Medical Univeristy (XT2018007)

\section{Availability of data and materials}

The Additional file used and analysed during the current study are available from the corresponding author on reasonable request.

\section{Ethics approval}

All animal experiments were approved by the Ethics Committee of Ningxia Medical University (document no. 2016-232), and carried out in accordance with the 2011 revised form of The Guide for the Care and Use of Laboratory Animals published by the U.S. National Institutes of Health.

\section{Consent for publication}

Not applicable.

\section{Competing interests}

The authors declare that they have no competing interests.

\section{Author details}

${ }^{1}$ Department of Pathogenic Biology and Medical Immunology, School of Basic Medical Sciences, Ningxia Medical University, Yinchuan 750004, Ningxia, China. ${ }^{2}$ Endocrinology Department, General Hospital of Ningxia Medical University, Yinchuan 750004, Ningxia, China. ${ }^{3}$ Endocrinology Department, People's Hospital of Ningxia Hui Autonomous Region, Yinchuan 750002, Ningxia, China. ${ }^{4}$ Clinical Medical College, Ningxia Medical University, Yinchuan 750004, Ningxia, China. ${ }^{5}$ College of Traditional Chinese Medicine, Ningxia Medical University, Yinchuan 750004, Ningxia, China.

Received: 4 August 2019 Accepted: 9 December 2019 Published online: 07 February 2020

\section{References}

1. Cho NH, Shaw JE, Karuranga S, Huang Y, Da RFJ, Ohlrogge AW, Malanda B. IDF diabetes atlas: global estimates of diabetes prevalence for 2017 and projections for 2045. Diabetes Res Clin Pract. 2018;138:271-81.

2. Brunkwall $\mathrm{L}$, Orho-Melander $\mathrm{M}$. The gut microbiome as a target for prevention and treatment of hyperglycaemia in type 2 diabetes: from current human evidence to future possibilities. DIABETOLOGIA. 2017;60(6):943-51.

3. Li K, Zhang L, Xue J, Yang X, Dong X, Sha L, Lei H, Zhang X, Zhu L, Wang Z, et al. Dietary inulin alleviates diverse stages of type 2 diabetes mellitus via anti-inflammation and modulating gut microbiota in $\mathrm{db} / \mathrm{db}$ mice. Food Funct. 2019:10(4):1915-27.

4. Larsen N, Vogensen FK, van den Berg FW, Nielsen DS, Andreasen AS, Pedersen BK, Al-Soud WA, Sorensen SJ, Hansen LH, Jakobsen M. Gut microbiota in human adults with type 2 diabetes differs from non-diabetic adults. PLoS One. 2010;5(2):e9085.

5. Zhang Q, Yu H, Xiao X, Hu L, Xin F, Yu X. Inulin-type fructan improves diabetic phenotype and gut microbiota profiles in rats. PEERJ. 2018;6:e4446.

6. Bouter KE, van Raalte DH, Groen AK, Nieuwdorp M. Role of the gut microbiome in the pathogenesis of obesity and obesity-related metabolic dysfunction. GASTROENTEROLOGY. 2017;152(7):1671-8.

7. Delzenne NM, Cani PD, Everard A, Neyrinck AM, Bindels LB. Gut microorganisms as promising targets for the management of type 2 diabetes. DIABETOLOGIA. 2015;58(10):2206-17.

8. Moura-Assis A, Afonso MS, de Oliveira V, Morari J, Dos SG, Koike M, Lottenberg AM, Ramos CR, Velloso LA, Sanchez RDSA, et al. Flaxseed oil rich in omega-3 protects aorta against inflammation and endoplasmic reticulum stress partially mediated by GPR120 receptor in obese, diabetic and dyslipidemic mice models. J Nutr Biochem. 2018:53:9-19.

9. Nie Q, Chen H, Hu J, Fan S, Nie S. Dietary compounds and traditional Chinese medicine ameliorate type 2 diabetes by modulating gut microbiota. Crit Rev Food Sci Nutr. 2019;59(6):848-63.

10. Conlon MA, Bird AR. The impact of diet and lifestyle on gut microbiota and human health. NUTRIENTS. 2014;7(1):17-44.

11. Hu JL, Nie SP, Xie MY. Antidiabetic mechanism of dietary polysaccharides based on their gastrointestinal functions. J Agric Food Chem. 2018;66(19):4781-6.

12. Ulven T, Christiansen E. Dietary fatty acids and their potential for controlling metabolic diseases through activation of FFA4/GPR120. Annu Rev Nutr. 2015:35:239-63.

13. White PJ, Marette A. Potential role of omega-3-derived resolution mediators in metabolic inflammation. Immunol Cell Biol. 2014:92(4):324-30.

14. Bang HO, Dyerberg J, Sinclair HM. The composition of the Eskimo food in north western Greenland. Am J Clin Nutr. 1980;33(12):2657-61.

15. Soleimani Z, Hashemdokht F, Bahmani F, Taghizadeh M, Memarzadeh MR, Asemi Z. Clinical and metabolic response to flaxseed oil omega-3 fatty acids supplementation in patients with diabetic foot ulcer: a randomized, double-blind, placebo-controlled trial. J Diabetes Complicat. 2017;31(9):1394-400.

16. Liu Y, Chen F, Odle J, Lin X, Jacobi SK, Zhu H, Wu Z, Hou Y. Fish oil enhances intestinal integrity and inhibits TLR4 and NOD2 signaling pathways in weaned pigs after LPS challenge. J Nutr. 2012;142(11):2017-24.

17. Gaspar RC, Veiga CB, Bessi MP, Datilo MN, Sant'Ana MR, Rodrigues PB, de Moura LP, Da SA, Santos GA, Catharino RR, et al. Unsaturated fatty acids from flaxseed oil and exercise modulate GPR120 but not GPR40 in the liver of obese mice: a new anti-inflammatory approach. J Nutr Biochem. 2019;66:52-62

18. Jangale NM, Devarshi PP, Bansode SB, Kulkarni MJ, Harsulkar AM. Dietary flaxseed oil and fish oil ameliorates renal oxidative stress, protein glycation, and inflammation in streptozotocin-nicotinamide-induced diabetic rats. J Physiol Biochem. 2016;72(2):327-36

19. Masiello P, Broca CR, Roye M, Manteghetti M, Hillaire-Buys D, Novelli M, Ribes G. Experimental NIDDM: development of a new model in adult rats administered streptozotocin and nicotinamide. Diabetes. 1998:47(2):224-9

20. Yuan T, Chu C, Shi R, Cui T, Zhang X, Zhao Y, Shi X, Hui Y, Pan J, Qian R, et al. ApoE-dependent protective effects of Sesamol on high-fat dietinduced behavioral disorders: regulation of the microbiome-gut-brain Axis. J Agric Food Chem. 2019;67(22):6190-6201.

21. Zhang L, Wang Y, Xiayu X, Shi C, Chen W, Song N, Fu X, Zhou R, Xu YF, Huang $L$, et al. Altered gut microbiota in a mouse model of Alzheimer's disease. J Alzheimers Dis. 2017;60(4):1241-57.

22. Zhao L. The gut microbiota and obesity: from correlation to causality. Nat Rev Microbiol. 2013;11(9):639-47.

23. Papaccio G, Pisanti FA, Latronico MV, Ammendola E, Galdieri M. Multiple low-dose and single high-dose treatments with streptozotocin do not generate nitric oxide. J Cell Biochem. 2000;77(1):82-91.

24. Shah MA, Reanmongkol W, Radenahmad N, Khalil R, UI-Haq Z, Panichayupakaranant P. Anti-hyperglycemic and anti-hyperlipidemic effects of rhinacanthins-rich extract from Rhinacanthus nasutus leaves in nicotinamide-streptozotocin induced diabetic rats. Biomed Pharmacother. 2019:113:108702. 
25. Ghasemi A, Khalifi S, Jedi S. Streptozotocin-nicotinamide-induced rat model of type 2 diabetes (review). Acta Physiol Hung. 2014;101(4):408-20.

26. Taheri RS, Sarker M, Rahmat A, Alkahtani SA, Othman F. The effect of pomegranate fresh juice versus pomegranate seed powder on metabolic indices, lipid profile, inflammatory biomarkers, and the histopathology of pancreatic islets of Langerhans in streptozotocin-nicotinamide induced type 2 diabetic Sprague-Dawley rats. BMC Complement Altern Med. 2017;17(1):156.

27. Wang F, Zhu H, Hu M, Wang J, Xia H, Yang X, Yang L, Sun G. Perilla oil supplementation improves hypertriglyceridemia and gut Dysbiosis in diabetic KKAy mice. Mol Nutr Food Res. 2018;62(24):e1800299.

28. Hashemzadeh AA, Nasoohi N, Raygan F, Aghadavod E, Akbari E, Taghizadeh M, Memarzadeh MR, Asemi Z. Flaxseed oil supplementation improve gene expression levels of PPAR-gamma, LP(a), IL-1 and TNF-alpha in type 2 diabetic patients with coronary heart disease. LIPIDS. 2017;52(11):907-15.

29. Yang ZH, Miyahara H, Takemura S, Hatanaka A. Dietary saury oil reduces hyperglycemia and hyperlipidemia in diabetic KKAy mice and in dietinduced obese C57BL/6J mice by altering gene expression. LIPIDS. 2011;46(5):425-34.

30. Haliga R, Mocanu V, Paduraru I, Stoica B, Oboroceanu T, Luca V. Effects of dietary flaxseed supplementation on renal oxidative stress in experimental diabetes. Rev Med Chir Soc Med Nat lasi. 2009;113(4):1200-4

31. Badawy EA, Rasheed WI, Elias TR, Hussein J, Harvi M, Morsy S, Mahmoud Y. Flaxseed oil reduces oxidative stress and enhances brain monoamines release in streptozotocin-induced diabetic rats. Hum Exp Toxicol. 2015;34(11):1133-8.

32. Burcelin R, Garidou L, Pomie C. Immuno-microbiota cross and talk: the new paradigm of metabolic diseases. Semin Immunol. 2012;24(1):67-74.

33. Wen L, Duffy A. Factors influencing the gut microbiota, inflammation, and type 2 diabetes. J Nutr. 2017;147(7):1468S-75S.

34. Festa A, D'Agostino RJ, Howard G, Mykkanen L, Tracy RP, Haffner SM. Chronic subclinical inflammation as part of the insulin resistance syndrome: the insulin resistance atherosclerosis study (IRAS). Circulation. 2000;102(1):42-7.

35. Hotamisligil GS, Shargill NS, Spiegelman BM. Adipose expression of tumor necrosis factor-alpha: direct role in obesity-linked insulin resistance. SCIENCE. 1993;259(5091):87-91.

36. Saisho Y. Metformin and inflammation: its potential beyond glucoselowering effect. Endocr Metab Immune Disord Drug Targets. 2015;15(3): 196-205.

37. Morey M, O'Gaora P, Pandit A, Helary C. Hyperglycemia acts in synergy with hypoxia to maintain the pro-inflammatory phenotype of macrophages. PLoS One. 2019;14(8):e220577.

38. Zhang P, Smith R, Chapkin RS, McMurray DN. Dietary (n-3) polyunsaturated fatty acids modulate murine Th1/Th2 balance toward the Th2 pole by suppression of Th1 development. J Nutr. 2005;135(7):1745-51.

39. Merchant AT, Curhan GC, Rimm EB, Willett WC, Fawzi WW. Intake of n-6 and $\mathrm{n}^{-3}$ fatty acids and fish and risk of community-acquired pneumonia in US men. Am J Clin Nutr. 2005;82(3):668-74.

40. Jangale NM, Devarshi PP, Dubal AA, Ghule AE, Koppikar SJ, Bodhankar SL, Chougale AD, Kulkarni MJ, Harsulkar AM. Dietary flaxseed oil and fish oil modulates expression of antioxidant and inflammatory genes with alleviation of protein glycation status and inflammation in liver of streptozotocin-nicotinamide induced diabetic rats. Food Chem 2013;141(1):187-95.

41. van der Poll $\mathrm{T}$, Jansen J, Levi $\mathrm{M}$, Ten $\mathrm{CH}$, Ten CJ, van Deventer SJ. Regulation of interleukin 10 release by tumor necrosis factor in humans and chimpanzees. J Exp Med. 1994;180(5):1985-8.

42. Brun P, Castagliuolo I, Di Leo V, Buda A, Pinzani M, Palu G, Martines D. Increased intestinal permeability in obese mice: new evidence in the pathogenesis of nonalcoholic steatohepatitis. Am J Physiol Gastrointest Liver Physiol. 2007;292(2):G518-25.

43. Martinez I, Muller CE, Walter J. Long-term temporal analysis of the human fecal microbiota revealed a stable core of dominant bacterial species. PLoS One. 2013:8(7):e69621.

44. Lambeth SM, Carson T, Lowe J, Ramaraj T, Leff JW, Luo L, Bell CJ, Shah VO. Composition, diversity and abundance of gut microbiome in Prediabetes and type 2 diabetes. J Diabetes Obes. 2015;2(3):1-7.

45. Kashtanova DA, Tkacheva ON, Doudinskaya EN, Strazhesko ID, Kotovskaya W, Popenko AS, Tyakht AV, Alexeev DG. Gut Microbiota in Patients with Different Metabolic Statuses: Moscow Study. Microorganisms. 2018:6(4). https://doi.org/10.3390/microorganisms6040098.
46. Egshatyan L, Kashtanova D, Popenko A, Tkacheva O, Tyakht A, Alexeev D, Karamnova N, Kostryukova E, Babenko V, Vakhitova M, et al. Gut microbiota and diet in patients with different glucose tolerance. Endocr Connect. 2016:5(1):1-9.

47. Kuang YS, Lu JH, Li SH, Li JH, Yuan MY, He JR, Chen NN, Xiao WQ, Shen SY, Qiu $L$, et al. Connections between the human gut microbiome and gestational diabetes mellitus. GIGASCIENCE. 2017;6(8):1-12.

48. Cummings JH, Pomare EW, Branch WJ, Naylor CP, Macfarlane GT. Short chain fatty acids in human large intestine, portal, hepatic and venous blood GUT. 1987;28(10):1221-7.

49. Chakraborti CK. New-found link between microbiota and obesity. World J Gastrointest Pathophysiol. 2015;6(4):110-9.

50. Costantini L, Molinari R, Farinon B, Merendino N. Impact of Omega-3 Fatty Acids on the Gut Microbiota. Int J Mol Sci. 2017;18(12).

51. Han L, Li T, Du M, Chang R, Zhan B, Mao X. Beneficial Effects of Potentilla discolor Bunge Water Extract on Inflammatory Cytokines Release and Gut Microbiota in High-Fat Diet and Streptozotocin-Induced Type 2 Diabetic Mice. Nutrients. 2019;11(3). https://doi.org/10.3390/nu11030670.

\section{Publisher's Note}

Springer Nature remains neutral with regard to jurisdictional claims in published maps and institutional affiliations.

\section{Ready to submit your research? Choose BMC and benefit from:}

- fast, convenient online submission

- thorough peer review by experienced researchers in your field

- rapid publication on acceptance

- support for research data, including large and complex data types

- gold Open Access which fosters wider collaboration and increased citations

- maximum visibility for your research: over $100 \mathrm{M}$ website views per year

At BMC, research is always in progress.

Learn more biomedcentral.com/submissions 